\title{
Shape coexistence in neutron-deficient krypton isotopes
}

E. Clément, ${ }^{1, *}$ A. Görgen, ${ }^{1}$ W. Korten, ${ }^{1}$ E. Bouchez, ${ }^{1}$ A. Chatillon, ${ }^{1, \dagger}$ J.-P. Delaroche,${ }^{2}$ M. Girod,${ }^{2}$ H. Goutte,${ }^{2}$ A. Hürstel, ${ }^{1}$ Y. Le Coz,${ }^{1,}$ A. Obertelli, ${ }^{1}$ S. Péru, ${ }^{2}$ Ch. Theisen, ${ }^{1}$ J. N. Wilson, ${ }^{1, \S}$ M. Zielińska, ${ }^{1,3}$ C. Andreoiu, ${ }^{4, \|}$ F. Becker, ${ }^{5}$

P. A. Butler, ${ }^{4}$ J. M. Casandjian, ${ }^{6,9}$ W. N. Catford, ${ }^{7}$ T. Czosnyka, ${ }^{3,{ }^{* *}}$ G. de France, ${ }^{6}$ J. Gerl, ${ }^{5}$ R.-D. Herzberg, ${ }^{4}$ J. Iwanicki, ${ }^{3,4}$ D. G. Jenkins, ${ }^{4, \dagger}$ G. D. Jones, ${ }^{4}$ P. J. Napiorkowski, ${ }^{3}$ G. Sletten, ${ }^{8}$ and C. N. Timis ${ }^{7}$

${ }^{1}$ CEA Saclay, DAPNIA/SPhN, F-91191 Gif-sur-Yvette, France

${ }^{2}$ CEA/DIF, DPTA/SPN, B.P. 12, F-91680 Bruyères-le-Châtel, France

${ }^{3}$ Heavy Ion Laboratory, Warsaw University, Warsaw, PL-02097, Poland

${ }^{4}$ Oliver Lodge Laboratory, University of Liverpool, Liverpool, L69 7ZE, United Kingdom

${ }^{5}$ Gesellschaft für Schwerionenforschung, D-64291 Darmstadt, Germany

${ }^{6}$ GANIL, BP-5027, F-14076 Caen Cedex, France

${ }^{7}$ Department of Physics, University of Surrey, Guildford, GU2 7XH, United Kingdom

${ }^{8}$ Niels Bohr Institute, Blegdamsvej 17, DK-2100 Copenhagen $\emptyset$, Denmark

(Received 2 February 2007; published 10 May 2007)

\begin{abstract}
Shape coexistence in the light krypton isotopes was studied in two low-energy Coulomb excitation experiments using radioactive ${ }^{74} \mathrm{Kr}$ and ${ }^{76} \mathrm{Kr}$ beams from the SPIRAL facility at GANIL. The ground-state bands in both isotopes were populated up to the $8^{+}$state via multi-step Coulomb excitation, and several non-yrast states were observed. Large sets of matrix elements were extracted for both nuclei from the observed $\gamma$-ray yields. Diagonal matrix elements were determined by utilizing the reorientation effect. In both isotopes the spectroscopic quadrupole moments for the ground-state bands and the bands based on excited $0_{2}^{+}$states are found to have opposite signs. The experimental data are interpreted within a phenomenological two-band mixing model and model-independent quadrupole invariants are deduced for the relevant $0^{+}$states using the complete sets of matrix elements and the formalism of quadrupole sum rules. Configuration mixing calculations based on triaxial Hartree-Fock-Bogolyubov calculations with the Gogny D1S effective interaction have been performed and are compared both with the experimental results and with recent calculations using the Skyrme SLy6 effective interaction and the full generator-coordinate method restricted to axial shapes.
\end{abstract}

DOI: 10.1103/PhysRevC.75.054313

PACS number(s): 23.20.Js, 21.60.-n, 25.70.De, 27.50.+e

\section{INTRODUCTION}

The shape of an atomic nucleus is a fundamental property reflecting the spatial distribution of the nucleons. Closed-shell nuclei are always spherical in their ground state because all orientations in space of the nucleon orbitals are equally probable. In nuclei with open shells the occupation of certain shape-driving orbitals tends to polarize the nucleus. To first order, when describing the nuclear potential by a simple harmonic oscillator, the binding energy is independent of the sign of the elongation parameter, and compressed ellipsoidal (oblate) and elongated (prolate) shapes should be equally

\footnotetext{
*Present address: ISOLDE, CERN, CH-1211 Geneva, Switzerland.

${ }^{\dagger}$ Present address: Gesellschaft für Schwerionenforschung, D-64291 Darmstadt, Germany.

${ }^{\ddagger}$ Present address: DEN/DTN/SMTM, CEA Cadarache, F-13108 Saint-Paul-lez-Durance, France.

${ }^{\S}$ Present address: Institut de Physique Nucléaire, IN2P3-CNRS, F-91406 Orsay Cedex, France.

"Present address: Department of Physics, University of Guelph, Guelph, Ontario, N1G 2W1, Canada.

"Present address: CEA Saclay, DAPNIA/SAp, F-91191 Gif-surYvette, France.

** Deceased.

${ }^{\dagger}$ Present address: Department of Physics, University of York, Heslington, York YO10 5DD, UK.
}

probable [1]. Calculations performed with more realistic potentials show that prolate ground states are much more abundant. This tendency is also confirmed by experiments showing a strong dominance of prolate ground-state shapes. This observation can be related to the shell structure of nuclei and in particular to the strength of the spin-orbit term relative to the radial term of the nuclear potential [2]. Both experiments and theory show that the prolate dominance is particularly evident in heavy nuclei $(Z, N>50)$, where the shell structure has changed from a harmonic oscillator type to a Mayer-Jensen type with a high- $j$ intruder orbital in each major shell. In lighter nuclei $(Z, N<40)$ prolate and oblate solutions appear more evenly in the ground states.

Many neutron-deficient nuclei in the mass $A=70-80$ region, especially close to the $N=Z$ line, have a large quadrupole deformation in their ground state. In addition, oblate and prolate shapes are predicted to coexist within a very small energy range of a few hundred $\mathrm{keV}$. This shape coexistence is due to the competition of large shell gaps in the particle level scheme for both oblate and prolate deformation at proton/neutron numbers 34, 36, and 38. The neutron-deficient $\mathrm{Se}$ and $\mathrm{Kr}$ isotopes are hence ideal candidates for the study of shape polarization and shape-mixing properties. Deformed shell-model calculations predict that the $N=Z$ nuclei ${ }^{68} \mathrm{Se}$ and ${ }^{72} \mathrm{Kr}$ have oblate ground states with a prolate configuration coexisting at low excitation energy [3]. The situation is predicted to be inversed for heavier isotopes, where a prolate 
ground state is expected to coexist with an excited oblate configuration.

A first experimental indication of shape coexistence in even-even nuclei is the observation of a low-lying $0_{2}^{+}$state, which can be interpreted as the ground state of a different shape. If both $0^{+}$states are (intrinsically) deformed, one would expect two rotational bands related to the different shapes. If the configurations come close in energy, the wave functions of states of the same spin and parity can mix and cause a distortion of the rotational bands. Shape coexistence in light krypton isotopes was first suggested by Piercey et al. [4] to explain the irregularities in the ground-state bands at low spin. A metastable low-lying $0_{2}^{+}$state, i.e., a shape isomer, was first reported for ${ }^{74} \mathrm{Kr}[5,6]$. More recently, an isomeric $0_{2}^{+}$state was observed in ${ }^{72} \mathrm{Kr}$ [7], extending the systematics to the $N=Z$ line. The excitation energy of the $0_{2}^{+}$states is decreasing from ${ }^{78} \mathrm{Kr}$ to ${ }^{74} \mathrm{Kr}$ and then increasing again for ${ }^{72} \mathrm{Kr}$. The measured strength of the electric monopole transitions $\rho^{2}(E 0)$, however, is maximal for ${ }^{74} \mathrm{Kr}$. The mixing amplitudes of the wave functions were derived from the distortion at low spin of the otherwise regular rotational bands, and were also found maximal for ${ }^{74} \mathrm{Kr}$ [7]. These observations were interpreted as evidence for an inversion of the ground-state deformation with decreasing neutron number: ${ }^{78} \mathrm{Kr}$ and ${ }^{76} \mathrm{Kr}$ are assumed to be prolate in their ground state, while an oblate shape becomes the ground state of ${ }^{72} \mathrm{Kr}$. For ${ }^{74} \mathrm{Kr}$ the intrinsic states of opposite deformation are assumed to be almost degenerate, and the measured displacement of the two physical $0^{+}$states is mostly due to the repulsion of the strongly mixed states [7]. This shape coexistence scenario is conclusive, but it is only based on indirect measures of the nuclear shapes. Neither the sign of the deformation nor the mixing of the low-spins states have been determined experimentally.

Low-energy Coulomb excitation is a well-established method to study collective excitations in nuclei [8]. When two nuclei are passing each other on close trajectories, but without coming into the range of the nuclear interaction, collective states can be excited in a purely electromagnetic process. Since the interaction time is relatively long, several successive excitations are possible, populating states up to relatively high spins. From the measured (differential) Coulomb excitation cross section the corresponding electromagnetic matrix elements can be extracted. Diagonal matrix elements can be determined and the (intrinsic) shape parameters extracted via second-order interference terms in the excitation process. Sensitivity to the diagonal matrix elements comes for example from the reorientation effect, which is caused by second-order transitions between the magnetic substates of an excited state [9]. Coulomb excitation is thus the only method that can directly distinguish between different shapes of the nucleus and is ideally suited to verify the shape coexistence scenario in the light $\mathrm{Se}$ and $\mathrm{Kr}$ isotopes.

Until very recently Coulomb excitation experiments at low energy were limited to stable or very long-lived nuclei, both for projectile or target excitation. With the availability of radioactive ion beams (RIBs) from ISOL facilities, such experiments are now starting to become possible for projectile excitation of short-lived unstable nuclei. In-flight facilities cannot deliver pure low-energy radioactive beams with acceptable emittance and are therefore only suitable for intermediate-energy but not for low-energy Coulomb excitation experiments. For a precise determination of diagonal matrix elements it is necessary to measure the differential Coulomb excitation cross section (or the excitation probability) very accurately at safe energies well below the Coulomb barrier over a large range of scattering angles. A variation of the atomic number $Z$ of the target nucleus increases the sensitivity of the measurement. The SPIRAL facility [10] at GANIL (Caen, France) delivers high-quality RIBs and especially rare gases are produced with relatively high intensities. Two projectile Coulomb excitation experiments were performed with beams of ${ }^{74} \mathrm{Kr}$ and ${ }^{76} \mathrm{Kr}$, respectively. The high precision of the data allowed exploiting the reorientation effect for the first time with radioactive beams.

\section{EXPERIMENTAL DETAILS}

The radioactive ${ }^{74} \mathrm{Kr}$ and ${ }^{76} \mathrm{Kr}$ beams were produced at the SPIRAL facility [10] at GANIL by fragmentation of an intense primary beam of stable ${ }^{78} \mathrm{Kr}$ of $10^{12}$ particles per second at $68.5 \mathrm{~A} \mathrm{MeV}$ on a thick carbon production target. The radioactive species were extracted, ionized in an ECR source, and post-accelerated in the $K=265$ CIME cyclotron to $4.4 \mathrm{~A}$ $\mathrm{MeV}$ for ${ }^{76} \mathrm{Kr}$ and $4.7 \mathrm{~A} \mathrm{MeV}$ for ${ }^{74} \mathrm{Kr}$. The average secondary beam intensity was $5 \times 10^{5}$ and $10^{4}$ pps for ${ }^{76} \mathrm{Kr}$ and ${ }^{74} \mathrm{Kr}$, respectively. Due to the excellent mass separation of the CIME cyclotron isobaric contaminants are strongly suppressed; only in the case of ${ }^{74} \mathrm{Kr}$ a small ${ }^{74} \mathrm{Se}$ contamination of $\sim 1 \%$ was observed. The ${ }^{76} \mathrm{Kr}$ and ${ }^{74} \mathrm{Kr}$ projectiles were Coulomb excited on ${ }^{208} \mathrm{~Pb}$ targets of 0.9 and $1.0 \mathrm{mg} / \mathrm{cm}^{2}$ thickness, respectively. The production scheme of the radioactive beams and the experimental set-up are shown schematically in Fig. 1.

Both experiments used the same experimental set-up. The scattered projectiles and the recoiling target nuclei were detected in an annular, highly segmented double-sided silicon strip detector (DSSD) of $300 \mu \mathrm{m}$ thickness. The detector

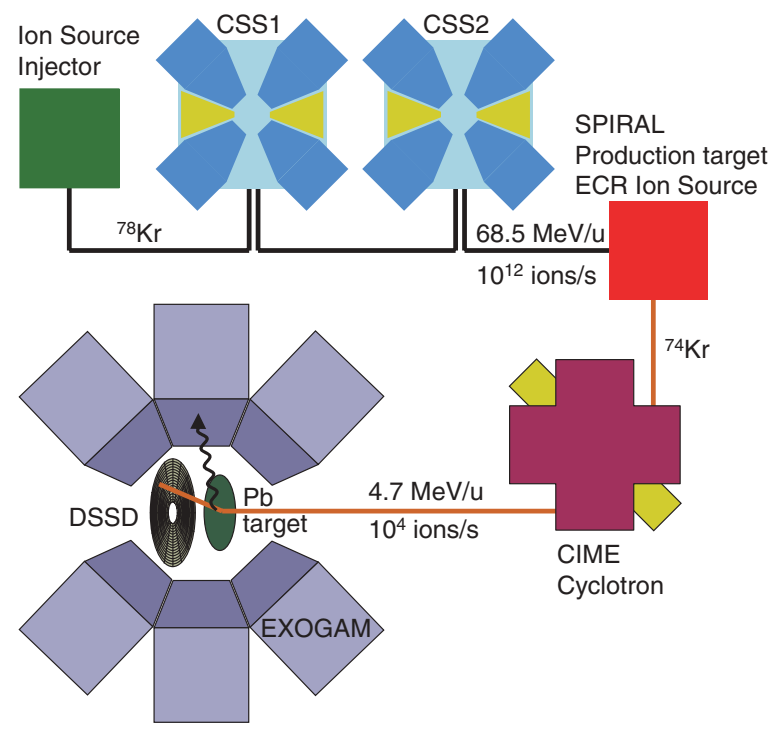

FIG. 1. (Color online) Production scheme of the radioactive ${ }^{74} \mathrm{Kr}$ and ${ }^{76} \mathrm{Kr}$ beams and schematic view of the detection set-up. 
was placed $25 \mathrm{~mm}$ downstream from the ${ }^{208} \mathrm{~Pb}$ target. The active area with inner and outer radii of 11 and $35 \mathrm{~mm}$, respectively, was segmented into 16 concentric rings and 16 azimuthal sectors. The energy resolution of the silicon detector was sufficient to distinguish between the scattered $\mathrm{Kr}$ projectiles and the recoiling $\mathrm{Pb}$ nuclei. This allowed a full kinematical reconstruction of the Coulomb excitation events when either the $\mathrm{Kr}$ or the $\mathrm{Pb}$ nucleus was detected. The DSSD covered scattering angles between $23.8^{\circ}$ and $54.5^{\circ}$ in the laboratory frame corresponding to a continuous range of scattering angles between $24^{\circ}$ and $145^{\circ}$ in the center-of-mass frame. For the scattering angles covered by the DSSD the distance of closest approach $d$ between projectile and target nuclei always corresponded to "safe" values to ensure a purely electromagnetic excitation, fulfilling the condition [11]

$$
d>1.25\left(A_{\mathrm{P}}^{1 / 3}+A_{\mathrm{T}}^{1 / 3}\right)+5 \mathrm{fm} .
$$

The segmentation of the silicon detector allowed measuring the differential Coulomb excitation cross section as a function of scattering angle. Unscattered projectiles left the target area through the central hole in the detector, reducing the radioactive background from the beam.

The $\gamma$ rays depopulating the Coulomb-excited states were detected in the EXOGAM array [12] of large segmented germanium clover detectors with escape suppression shields. Each clover detector comprises four individual germanium crystals, and each crystal is electrically segmented into four longitudinal segments. The array comprised six full-size and one smaller clover detector for the ${ }^{76} \mathrm{Kr}$ experiment, and seven large and four smaller detectors for the ${ }^{74} \mathrm{Kr}$ experiment. The detectors were placed at $90^{\circ}$ and $135^{\circ}$ with respect to the beam axis, and the distance between the front face of the detectors and the target was $11.2 \mathrm{~cm}$ for the large and $14 \mathrm{~cm}$ for the smaller detectors. The efficiency for full-energy absorption of a $1.3 \mathrm{MeV} \gamma$ ray was measured to be $12 \%$ during the ${ }^{74} \mathrm{Kr}$ experiment. Events were recorded when at least one $\gamma$ ray was detected in coincidence with one of the collision partners. The coincidence requirement suppressed the very large background from the radioactive beam almost completely. The segmentation of both the germanium and the silicon detectors allowed a precise determination of the relative angle between scattered $\mathrm{Kr}$ projectiles and the emitted $\gamma$ rays. After Doppler correction a resolution of $8 \mathrm{keV}$ was obtained for a $\gamma$ ray of $500 \mathrm{keV}$.

\section{DATA ANALYSIS AND RESULTS}

\section{A. ${ }^{76} \mathrm{Kr}$ Experiment}

The total Doppler corrected $\gamma$-ray spectrum in coincidence with either the scattered ${ }^{76} \mathrm{Kr}$ projectiles or the recoiling ${ }^{208} \mathrm{~Pb}$ target nuclei is shown in Fig. 2. The spectrum is very clean and neither background from the radioactive decay of the beam nor from isobaric contaminants of the beam are present. Data were collected for $\sim 50 \mathrm{~h}$ with a secondary beam intensity of $5 \times 10^{5} \mathrm{pps}$. The ground-state band was observed up to the $8^{+}$ state, populated in multi-step Coulomb excitation, and several non-yrast states were excited. A partial level scheme of ${ }^{76} \mathrm{Kr}$ is presented in Fig. 3, showing all states that were included in

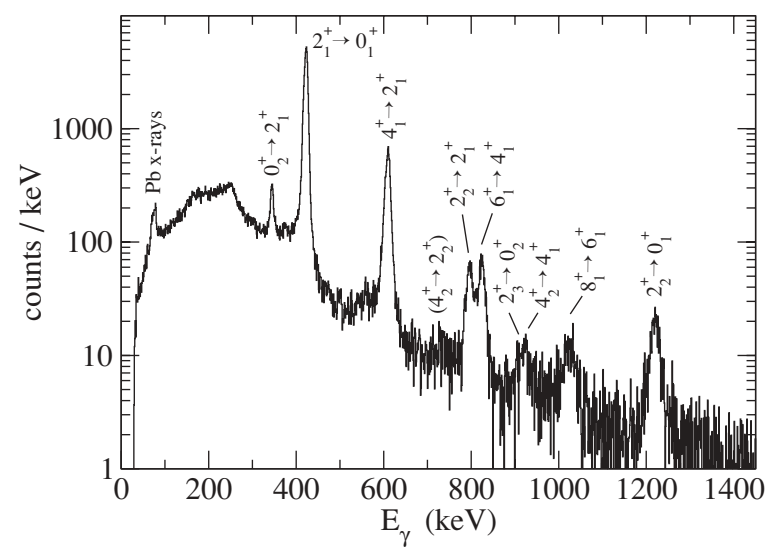

FIG. 2. Total $\gamma$-ray spectrum in logarithmic scale after Coulomb excitation of the $4.4 A \mathrm{MeV}^{76} \mathrm{Kr}$ beam on a ${ }^{208} \mathrm{~Pb}$ target of $0.9 \mathrm{mg} / \mathrm{cm}^{2}$ thickness in coincidence with either the scattered beam particle or recoiling target nucleus.

the Coulomb excitation analysis and all transitions that were observed. All states and transitions of the level scheme in Fig. 3 had been observed previously [13].

The $0_{2}^{+}$state at $770 \mathrm{keV}$, which is a candidate for having a shape different from that of the ground state, is populated and its decay to the $2_{1}^{+}$state observed. The $2_{3}^{+}$state at $1687 \mathrm{keV}$ is feeding the $0_{2}^{+}$state. However, this transition of $918 \mathrm{keV}$ is not fully resolved from the $4_{2}^{+} \rightarrow 4_{1}^{+}$transition with $923 \mathrm{keV}$. The $\Delta J=2$ sequence above the $2_{2}^{+}$state at $1222 \mathrm{keV}$ has been interpreted [14] as a $K=2$ quasi-gamma band together with a $\Delta J=2$ sequence on top of a $3^{+}$state at $1733 \mathrm{keV}$, which was not populated in the present Coulomb excitation experiment. The $2_{2}^{+}$state of this band, however, was populated and possibly also the $4_{2}^{+}$state, so that all even-spin members of the band have been included in the Coulomb excitation analysis.

To extract matrix elements from the differential Coulomb excitation cross sections and the observed $\gamma$-ray yields, the data was divided into several subsets corresponding to different ranges of scattering angles. Because the innermost rings and some rings in the center of the segmented silicon detector

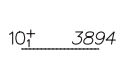

$4 \underline{068 \quad 10_{1}^{+}}$

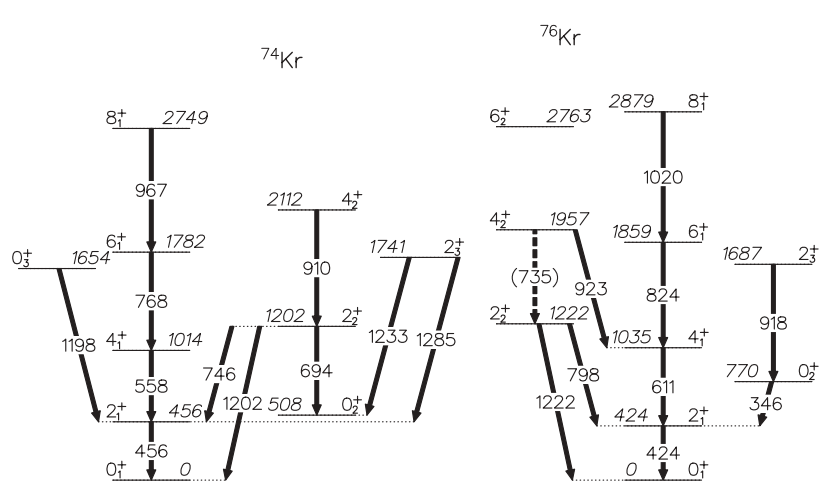

FIG. 3. Partial level schemes of ${ }^{74} \mathrm{Kr}$ (left) and ${ }^{76} \mathrm{Kr}$ (right) showing all transitions observed in the measurement and their energies in $\mathrm{keV}$ and all states that were included in the Coulomb excitation analysis. 
TABLE I. Observed $\gamma$-ray transitions in ${ }^{76} \mathrm{Kr}$ with their intensities (without efficiency correction) for four different ranges of center-of-mass scattering angles.

\begin{tabular}{|c|c|c|c|c|c|}
\hline Data set & $I_{i}^{\pi}$ & $I_{f}^{\pi}$ & $E_{\gamma}(\mathrm{keV})$ & Counts & Error \\
\hline A & $2_{1}^{+}$ & $0_{1}^{+}$ & 424 & 18426 & 190 \\
\hline \multirow[t]{5}{*}{$\left(39.5^{\circ}, 49.0^{\circ}\right)$} & $4_{1}^{+}$ & $2_{1}^{+}$ & 610 & 1122 & 50 \\
\hline & $6_{1}^{+}$ & $4_{1}^{+}$ & 825 & 41 & 9 \\
\hline & $2_{2}^{+}$ & $2_{1}^{+}$ & 797 & 132 & 16 \\
\hline & $2_{2}^{+}$ & $0_{1}^{+}$ & 1221 & 77 & 14 \\
\hline & $0_{2}^{+}$ & $2_{1}^{+}$ & 346 & 154 & 40 \\
\hline B & $2_{1}^{+}$ & $0_{1}^{+}$ & 424 & 11595 & 140 \\
\hline \multirow[t]{5}{*}{$\left(61.4^{\circ}, 71.8^{\circ}\right)$} & $4_{1}^{+}$ & $2_{1}^{+}$ & 610 & 2141 & 61 \\
\hline & $6_{1}^{+}$ & $4_{1}^{+}$ & 825 & 171 & 18 \\
\hline & $2_{2}^{+}$ & $2_{1}^{+}$ & 797 & 211 & 18 \\
\hline & $2_{2}^{+}$ & $0_{1}^{+}$ & 1221 & 113 & 15 \\
\hline & $0_{2}^{+}$ & $2_{1}^{+}$ & 346 & 314 & 35 \\
\hline $\mathrm{C}$ & $2_{1}^{+}$ & $0_{1}^{+}$ & 424 & 14123 & 168 \\
\hline \multirow[t]{8}{*}{$\left(71.0^{\circ}, 87.7^{\circ}\right)$} & $4_{1}^{+}$ & $2_{1}^{+}$ & 610 & 3343 & 82 \\
\hline & $6_{1}^{+}$ & $4_{1}^{+}$ & 825 & 503 & 31 \\
\hline & $8_{1}^{+}$ & $6_{1}^{+}$ & 1019 & 91 & 14 \\
\hline & $2_{2}^{+}$ & $2_{1}^{+}$ & 797 & 413 & 29 \\
\hline & $2_{2}^{+}$ & $0_{1}^{+}$ & 1221 & 203 & 20 \\
\hline & $0_{2}^{+}$ & $2_{1}^{+}$ & 346 & 706 & 51 \\
\hline & $2_{3}^{+}$ & $0_{2}^{+}$ & $918^{\mathrm{a}}$ & \multirow[t]{2}{*}{81} & \multirow[t]{2}{*}{18} \\
\hline & $4_{2}^{+}$ & $4_{1}^{+}$ & $923^{\mathrm{a}}$ & & \\
\hline $\mathrm{D}$ & $2_{1}^{+}$ & $0_{1}^{+}$ & 424 & 5924 & 83 \\
\hline \multirow[t]{8}{*}{$\left(107.0^{\circ}, 121.5^{\circ}\right)$} & $4_{1}^{+}$ & $2_{1}^{+}$ & 610 & 2308 & 68 \\
\hline & $6_{1}^{+}$ & $4_{1}^{+}$ & 825 & 493 & 50 \\
\hline & $8_{1}^{+}$ & $6_{1}^{+}$ & 1019 & 115 & 21 \\
\hline & $2_{2}^{+}$ & $2_{1}^{+}$ & 797 & 251 & 40 \\
\hline & $2_{2}^{+}$ & $0_{1}^{+}$ & 1221 & 177 & 34 \\
\hline & $0_{2}^{+}$ & $2_{1}^{+}$ & 346 & 789 & 75 \\
\hline & $2_{3}^{+}$ & $0_{2}^{+}$ & $918^{\mathrm{a}}$ & 118 & 23 \\
\hline & $4_{2}^{+}$ & $4_{1}^{+}$ & $923^{\mathrm{a}}$ & & \\
\hline
\end{tabular}

${ }^{a}$ Unresolved doublet; yield of the sum of both transitions.

were not functioning properly, only a partial range of scattering angles covered by the detector was used in the analysis. This is illustrated in Fig. 4, which shows the cross section to populate the states of the ground-state band as a function of scattering angle (in the center-of-mass frame). The ranges that were used in the analysis are indicated by the vertical lines and are given in Table I. The excitation probability for the small scattering angles, where the cross section to populate the $2_{1}^{+}$state is

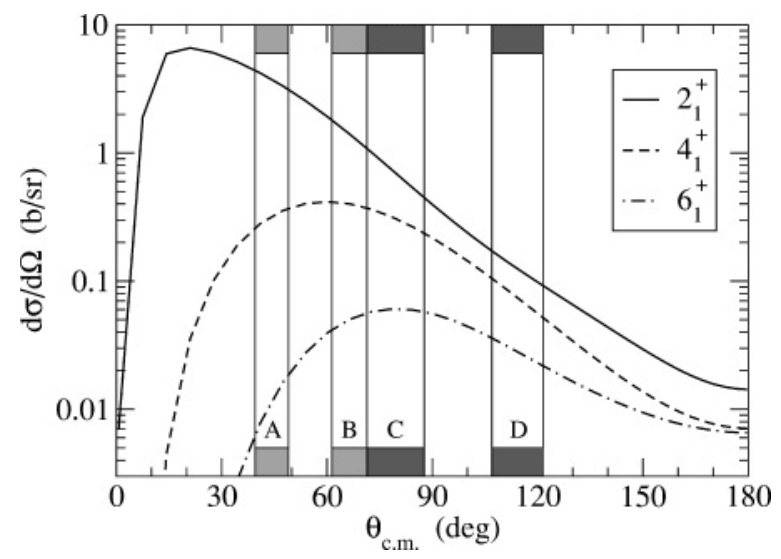

FIG. 4. Differential Coulomb excitation cross section to populate the $2_{1}^{+}, 4_{1}^{+}$, and $6_{1}^{+}$states in ${ }^{76} \mathrm{Kr}$. The angular ranges (in the center-ofmass system) covered by the working rings of the segmented silicon detector are labeled A-D.

largest, could not be measured. However, the ranges where the higher-lying states are populated with the highest cross sections are covered by the detector. Moreover, the sensitivity to higher-order effects, which allow determining the diagonal matrix elements, comes mostly from the large scattering angles. The division of the total data into the four ranges shown in Fig. 4 was found to be a good compromise between the largest possible number of data subsets for different angular ranges and the minimum level of statistics required to extract the $\gamma$-ray yields from the individual spectra.

The $\gamma$-ray spectra for the four data sets are shown in Fig. 5 and the $\gamma$-ray yields extracted from these spectra are summarized in Table I. As the center-of-mass scattering angle is increasing for the data sets from $\mathrm{A}$ to $\mathrm{D}$, the impact parameter and the distance of closest approach are decreasing. As a consequence, the probability to populate states at higher excitation energy and higher angular momentum via multi-step excitation is strongly enhanced for large scattering angles. This is illustrated in Fig. 6, where the $\gamma$-ray yields are plotted as functions of the scattering angle for several transitions normalized to the $2_{1}^{+} \rightarrow 0_{1}^{+}$transition. The $\gamma$-ray yields show not only a strong dependence on the scattering angle, but this dependence also differs significantly for the different transitions, which illustrates the sensitivity of the data to the matrix elements.

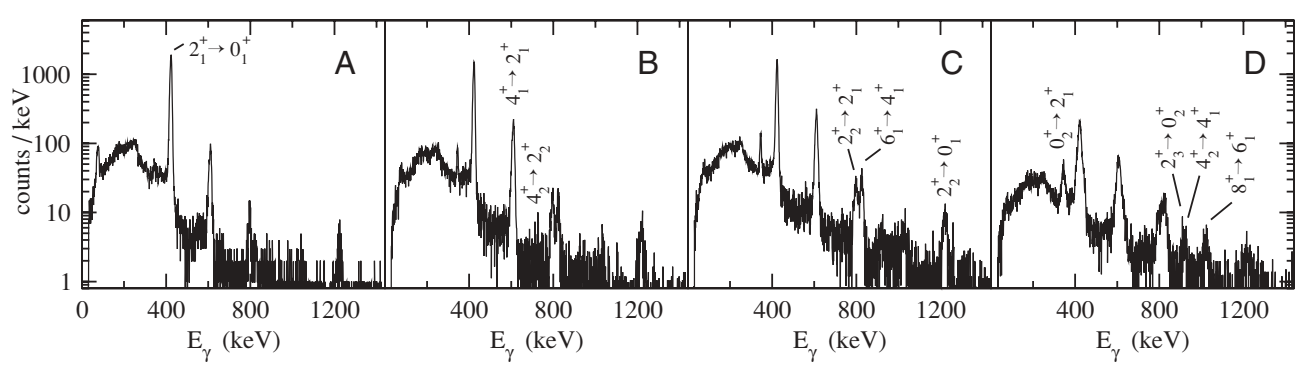

FIG. 5. Spectra after Coulomb excitation of ${ }^{76} \mathrm{Kr}$ on ${ }^{208} \mathrm{~Pb}$ for the four subsets of data corresponding to different ranges of scattering angles as defined in Table I. 


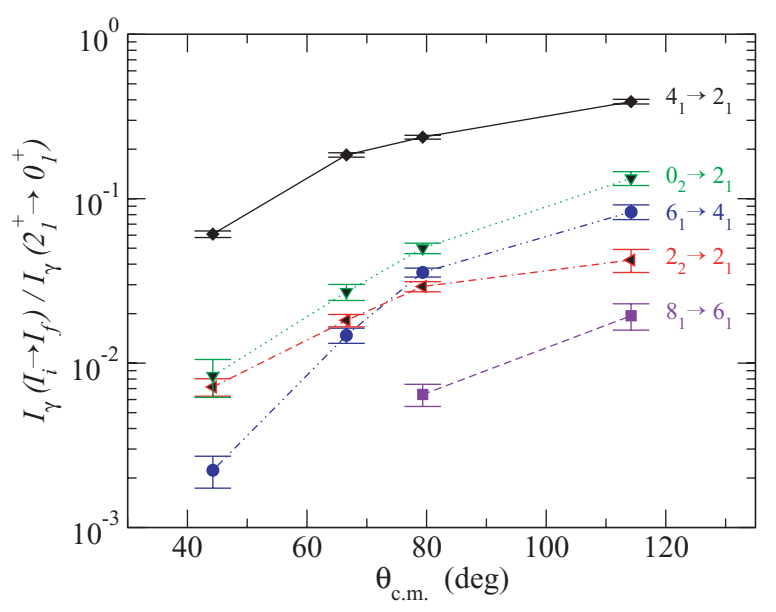

FIG. 6. (Color online) Intensity of some of the $\gamma$-ray transitions in ${ }^{76} \mathrm{Kr}$ as a function of scattering angle in the center-of-mass frame. All intensities have been normalized to the $2_{1}^{+} \rightarrow 0_{1}^{+}$transition.

\section{B. ${ }^{74} \mathrm{Kr}$ Experiment}

The production rate of ${ }^{74} \mathrm{Kr}$ with SPIRAL is at the limit of feasibility for a measurement of spectroscopic quadrupole moments with the low-energy Coulomb excitation technique utilizing the reorientation effect. An average secondary beam intensity of $10^{4}$ pps was achieved during the experiment. The lower beam intensity compared to the ${ }^{76} \mathrm{Kr}$ experiment was partly compensated by a longer running time of $\sim 150 \mathrm{~h}$. Furthermore, the experimental difficulties concerning the silicon detector could be resolved and a larger range of scattering angles was covered. The number of germanium clover detectors in the EXOGAM array was also increased, resulting in a higher full-energy detection efficiency of $12 \%$ at a $\gamma$-ray energy of $1.3 \mathrm{MeV}$. Even though the secondary beam intensity for ${ }^{74} \mathrm{Kr}$ was 50 times smaller compared to the ${ }^{76} \mathrm{Kr}$ experiment, the level of statistics was only reduced by a factor of five. The total $\gamma$-ray spectrum in coincidence with either scattered ${ }^{74} \mathrm{Kr}$ projectiles or recoiling target nuclei is shown in Fig. 7. In addition to transitions in ${ }^{74} \mathrm{Kr}$ the $2_{1}^{+} \rightarrow 0_{1}^{+}$transition

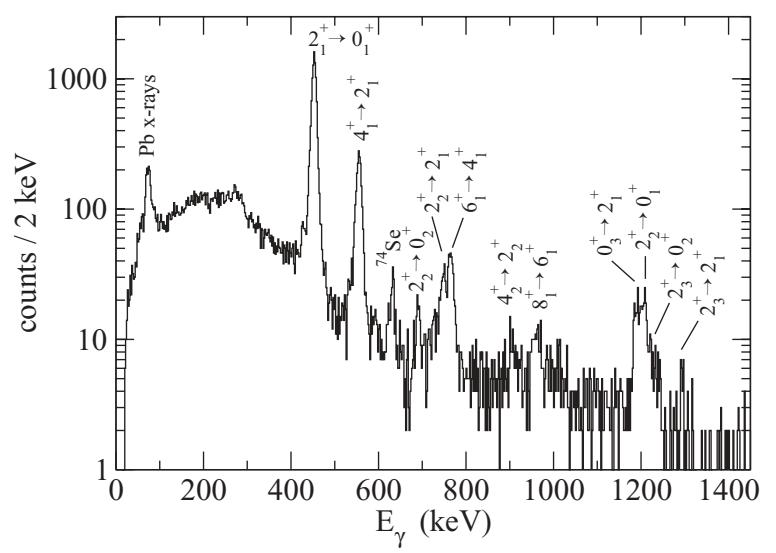

FIG. 7. Total $\gamma$-ray spectrum after Coulomb excitation of the $4.7 \mathrm{~A} \mathrm{MeV}{ }^{74} \mathrm{Kr}$ beam on a ${ }^{208} \mathrm{~Pb}$ target of $1.0 \mathrm{mg} / \mathrm{cm}^{2}$ thickness in coincidence with either the scattered beam particle or recoiling target nucleus. of ${ }^{74} \mathrm{Se}$ is also visible in the spectrum. Its strength accounts for $1.2 \%$ of the total beam intensity. No other contaminants of the beam were observed.

A partial level scheme of ${ }^{74} \mathrm{Kr}$ with the observed transitions is presented in Fig. 3. As was the case for ${ }^{76} \mathrm{Kr}$, the ground-state band of ${ }^{74} \mathrm{Kr}$ was populated up to the $8^{+}$state. The metastable $0_{2}^{+}$state at $508 \mathrm{keV}$ was interpreted as a shape isomer corresponding to a shape different from that of the ground state [7]. This state is populated via the $694 \mathrm{keV}$ transition from the $2_{2}^{+}$state. Its decay proceeds via an enhanced $E 0$ transition to the ground state and a strongly converted $E 2$ transition of $52 \mathrm{keV}$ to the $2_{1}^{+}$state [5-7] and is therefore not observed in this experiment. The $2_{2}^{+}$state also decays to the $2_{1}^{+}$and directly to the ground state. The branching ratio for the decay of the $2_{2}^{+}$ state is known from an earlier measurement [15]. A $4_{2}^{+}$state is expected above the $2_{2}^{+}$state as part of a rotational structure, but has not been reported previously. A new transition is observed at $910 \mathrm{keV}$ in the spectrum of Fig. 7, which does not correspond to any known transition in ${ }^{74} \mathrm{Kr}$ or neighboring nuclei that could potentially contaminate the beam. Because the energy and, as will be shown, the matrix element of this transition agree with the expected rotational state, a $4_{2}^{+}$ state is tentatively placed at $2112 \mathrm{keV}$. Weak $\gamma \gamma$ coincidence data were obtained confirming the above assignment, but an alternative interpretation of the $910 \mathrm{keV}$ peak as a $4_{2}^{+} \rightarrow 4_{1}^{+}$ transition cannot be completely excluded. In this case the $4_{2}^{+}$ state would be located $188 \mathrm{keV}$ lower.

A third $0^{+}$state at $1654 \mathrm{keV}$ and a third $2^{+}$state at $1741 \mathrm{keV}$ excitation energy have been observed after $\beta$ decay [15]. The $0_{3}^{+} \rightarrow 2_{1}^{+}$transition of $1198 \mathrm{keV}$ is not resolved from the $2_{2}^{+} \rightarrow 0_{1}^{+}$transition of $1202 \mathrm{keV}$. The larger width of the peak at $1200 \mathrm{keV}$, however, shows that the $0_{3}^{+}$state was also populated. The $2_{3}^{+} \rightarrow 0_{2}^{+}$transition of $1233 \mathrm{keV}$ is visible as a shoulder of this peak, and a very weak transition at $1285 \mathrm{keV}$ is interpreted as the $2_{3}^{+} \rightarrow 2_{1}^{+}$transition.

The full data set for ${ }^{74} \mathrm{Kr}$ was divided into four ranges of scattering angles as shown in Table II. The first two ranges, $\mathrm{A}$ and $\mathrm{B}$, correspond to the detection of the ${ }^{74} \mathrm{Kr}$ projectile in the silicon detector, whereas the last two ranges, $\mathrm{C}$ and $\mathrm{D}$, correspond to the detection of the recoiling ${ }^{208} \mathrm{~Pb}$ nuclei from the target. The individual spectra from the four subsets of data are shown in Fig. 8. This division is again a compromise between the maximum number of data sets and the minimum level of statistics required to extract the $\gamma$-ray yields. A division into only two ranges of scattering angles improves the uncertainty of the $\gamma$-ray yields, but was found to result in an insufficient number of data points to determine the large number of matrix elements needed to describe the data. The yields extracted from the spectra of Fig. 8 are summarized in Table II.

During the data analysis it was found that the silicon detector was not fully aligned with the beam axis in the experiment. The count rates are not isotropically distributed over the azimuthal sectors of the detector. By measuring the Rutherford scattering cross section individually for the azimuthal sectors and comparing to a Monte Carlo simulation, the displacement of the detector with respect to the beam axis was found to be $3.0(5) \mathrm{mm}$. This misalignment breaks the cylindrical symmetry of the set-up and introduces an azimuthal 
TABLE II. Observed $\gamma$-ray transitions in ${ }^{74} \mathrm{Kr}$ with their intensities (without efficiency correction) for the four different ranges of scattering angles.

\begin{tabular}{|c|c|c|c|c|c|}
\hline Data set & $I_{i}$ & $I_{f}$ & $E_{\gamma}(\mathrm{keV})$ & Counts & Error \\
\hline A & $2_{1}^{+}$ & $0_{1}^{+}$ & 456 & 4550 & 200 \\
\hline \multirow[t]{7}{*}[24.0^{\circ},54.5^{\circ}]{} & $4_{1}^{+}$ & $2_{1}^{+}$ & 558 & 400 & 80 \\
\hline & $6_{1}^{+}$ & $4_{1}^{+}$ & 768 & 27 & 10 \\
\hline & $8_{1}^{+}$ & $6_{1}^{+}$ & 967 & 11 & 6 \\
\hline & $2_{2}^{+}$ & $2_{1}^{+}$ & 746 & 36 & 6 \\
\hline & $0_{3}^{+}$ & $2_{1}^{+}$ & $1198^{a}$ & \multirow{2}{*}{82} & \multirow{2}{*}{10} \\
\hline & $2_{2}^{+}$ & $0_{1}^{+}$ & $1202^{\mathrm{a}}$ & & \\
\hline & $2_{2}^{+}$ & $0_{2}^{+}$ & 694 & 26 & 5 \\
\hline B & $2_{1}^{+}$ & $0_{1}^{+}$ & 456 & 2044 & 100 \\
\hline \multirow[t]{8}{*}[54.5^{\circ},73.9^{\circ}]{} & $4_{1}^{+}$ & $2_{1}^{+}$ & 558 & 445 & 30 \\
\hline & $6_{1}^{+}$ & $4_{1}^{+}$ & 768 & 55 & 10 \\
\hline & $8_{1}^{+}$ & $6_{1}^{+}$ & 967 & 15 & 5 \\
\hline & $2_{2}^{+}$ & $2_{1}^{+}$ & 746 & 55 & 10 \\
\hline & $0_{3}^{+}$ & $2_{1}^{+}$ & $1198^{a}$ & \multirow{2}{*}{55} & \multirow{2}{*}{15} \\
\hline & $2_{2}^{+}$ & $0_{1}^{+}$ & $1202^{\mathrm{a}}$ & & \\
\hline & $2_{2}^{+}$ & $0_{2}^{+}$ & 694 & 22 & 5 \\
\hline & $2_{3}^{+}$ & $0_{2}^{+}$ & 1233 & 17 & 10 \\
\hline $\mathrm{C}$ & $2_{1}^{+}$ & $0_{1}^{+}$ & 456 & 1775 & 100 \\
\hline \multirow[t]{10}{*}[67.1^{\circ},97.3^{\circ}]{} & $4_{1}^{+}$ & $2_{1}^{+}$ & 558 & 630 & 50 \\
\hline & $6_{1}^{+}$ & $4_{1}^{+}$ & 768 & 140 & 25 \\
\hline & $8_{1}^{+}$ & $6_{1}^{+}$ & 967 & 35 & 20 \\
\hline & $2_{2}^{+}$ & $2_{1}^{+}$ & 746 & 103 & 15 \\
\hline & $0_{3}^{+}$ & $2_{1}^{+}$ & $1198^{\mathrm{a}}$ & \multirow{2}{*}{112} & \multirow{2}{*}{10} \\
\hline & $2_{2}^{+}$ & $0_{1}^{+}$ & $1202^{\mathrm{a}}$ & & \\
\hline & $2_{2}^{+}$ & $0_{2}^{+}$ & 694 & 35 & 15 \\
\hline & $2_{3}^{+}$ & $0_{2}^{+}$ & 1233 & 25 & 10 \\
\hline & $\left(4_{2}^{+}\right)$ & $2_{2}^{+b}$ & 910 & 8 & 5 \\
\hline & $2_{3}^{+}$ & $2_{1}^{+}$ & 1285 & 16 & 5 \\
\hline $\mathrm{D}$ & $2_{1}^{+}$ & $0_{1}^{+}$ & 456 & 1090 & 100 \\
\hline \multirow{10}{*}[97.3^{\circ},144.5^{\circ}]{} & $4_{1}^{+}$ & $2_{1}^{+}$ & 558 & 440 & 30 \\
\hline & $6_{1}^{+}$ & $4_{1}^{+}$ & 768 & 130 & 30 \\
\hline & $8_{1}^{+}$ & $6_{1}^{+}$ & 967 & 53 & 20 \\
\hline & $2_{2}^{+}$ & $2_{1}^{+}$ & 746 & 90 & 30 \\
\hline & $0_{3}^{+}$ & $2_{1}^{+}$ & $1198^{\mathrm{a}}$ & \multirow{2}{*}{59} & \multirow{2}{*}{15} \\
\hline & $2_{2}^{+}$ & $0_{1}^{+}$ & $1202^{\mathrm{a}}$ & & \\
\hline & $2_{2}^{+}$ & $0_{2}^{+}$ & 694 & 25 & 15 \\
\hline & $2_{3}^{+}$ & $0_{2}^{+}$ & 1233 & 25 & 15 \\
\hline & $\left(4_{2}^{+}\right)$ & $2_{2}^{+b}$ & 910 & 8 & 4 \\
\hline & $2_{3}^{+}$ & $2_{1}^{+}$ & 1285 & 12 & 4 \\
\hline
\end{tabular}

anresolved doublet; yield of the sum of both transitions.

${ }^{\mathrm{b}}$ Alternative assignment $\left(4_{2}^{+}\right) \rightarrow 4_{1}^{+}$.

dependence of the scattering angle $\theta(\phi)$, which had to be taken into account for the Doppler correction of the $\gamma$ rays and the Coulomb excitation analysis. The small overlap between the ranges of scattering angles $\mathrm{B}$ and $\mathrm{C}$ (see Table II) is also due to this misalignment. The Doppler correction improves significantly when taking the displacement into account, and the same resolution as for the ${ }^{76} \mathrm{Kr}$ experiment is achieved. This is shown in Fig. 9, where the different steps of the Doppler correction process are illustrated.

\section{COULOMB EXCITATION ANALYSIS}

\section{A. GOSIA analysis}

The Coulomb excitation analysis was performed using the least squares fitting code GOSIA $[8,16]$. A standard $\chi^{2}$ function is constructed from the measured $\gamma$-ray yields and those calculated from a complete set of electromagnetic matrix elements, both transitional and diagonal, between all known states involved in the excitation process (see Fig. 3). As long as the "safe" condition from Eq. (1) is fulfilled, the electromagnetic excitation probability can be calculated with very high precision in a semiclassical way [9]. The ${ }^{208} \mathrm{~Pb}$ target is assumed to be inert and its electromagnetic excitation can be neglected due to the high excitation energy of the first excited state $\left(I^{\pi}=3^{-}\right.$at $\left.2.6 \mathrm{MeV}\right)$.

To exploit the dependence of the excitation probability on the scattering angle, the data is divided into several subsets corresponding to different ranges of scattering angles, as described in the previous section. An alternative (or additional) method would be to use different target materials and exploit the $Z$ dependence of the Coulomb excitation cross section. Due to the weak intensities of the radioactive beams, the level of statistics of the $\gamma$-ray spectra is limited, and the bins of scattering angles are relatively wide. As the number of matrix elements, i.e., the number of degrees of freedom in the fitting process, is similar to the number of data points ( $\gamma$-ray yields), the fitting problem is underdetermined. However, the convergence of the fit can be improved by using experimentally known spectroscopic data such as lifetimes, branching and mixing ratios as additional data points in the fit. The experimental uncertainties of the respective spectroscopic information enter into the $\chi^{2}$ fit of the matrix elements. In experiments with stable beams, which do not suffer from low statistics, these quantities can be treated as free parameters, and all the spectroscopic information can in principle be extracted directly from the Coulomb excitation data.

To calculate the $\gamma$-ray yields from the matrix elements, the position and geometry of all detectors and their relative efficiency have to be taken into account. In view of the close geometry it is also important to correct for the small displacement of the silicon detector with respect to the beam axis in the ${ }^{74} \mathrm{Kr}$ experiment. The exact reproduction of the experimental yields requires the integration over the range of scattering angles as defined in Tables I and II, and over the range of bombarding energies resulting from the energy loss of the projectiles in the lead target. The $\gamma$-ray intensities are corrected for internal conversion. The angular distribution of the $\gamma$ rays is taken into account; it is corrected for relativistic effects and the attenuation caused by the nuclear deorientation effect during recoil into vacuum.

It is of great advantage that the level schemes of both ${ }^{74} \mathrm{Kr}$ and ${ }^{76} \mathrm{Kr}$ were known and that all known transitions could be identified. Therefore most of the matrix elements 


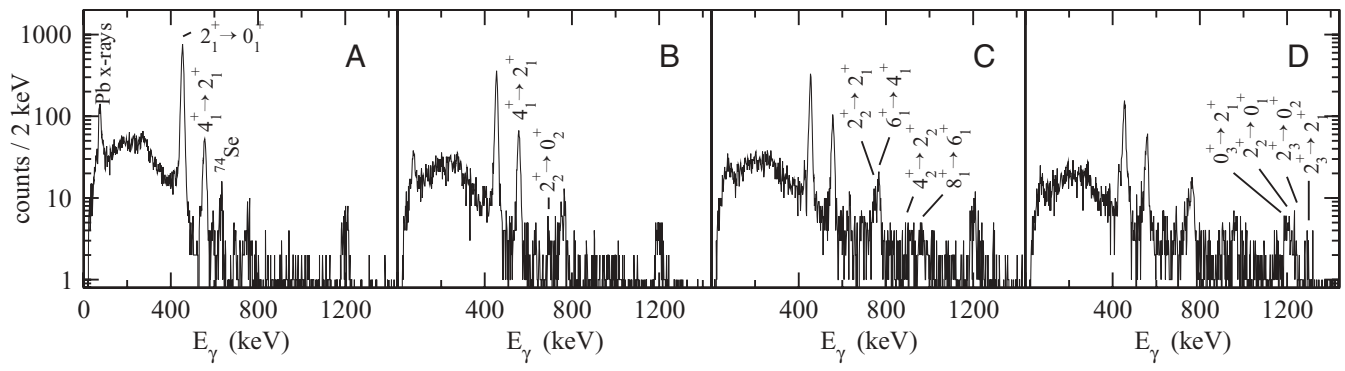

FIG. 8. Spectra from the ${ }^{74} \mathrm{Kr}$ experiment corresponding to the different ranges of scattering angles as defined in Table II.

that are important in the excitation process are well defined. The non-observation of a transition, i.e., an upper limit of its intensity, is also used in the fitting process and has to be reproduced by the matrix elements. In some cases it is possible to extract $E 2$ matrix elements between known states even if no transition was observed. The nuclear levels are first grouped into band structures and the matrix elements are initialized assuming a rotational relation between them. This serves only the purpose of finding realistic starting values. No assumptions concerning the rotational structure of the states are made during the minimization process.

The result of the $\chi^{2}$ minimization is a set of reduced matrix elements $\left\langle I_{2}\|\mathcal{M}(E 2)\| I_{1}\right\rangle$ that reproduces the experimental

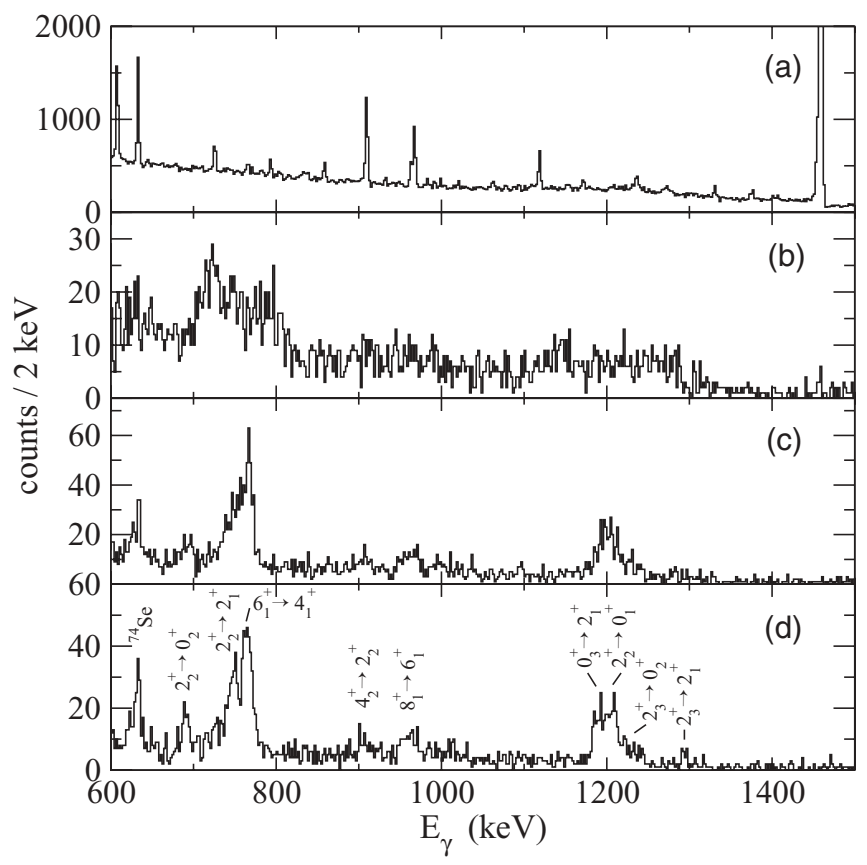

FIG. 9. Gamma-ray spectra from the ${ }^{74} \mathrm{Kr}$ experiment illustrating the data processing: Spectrum (a) has no condition on the particle detector; only the radioactive background from the beam and the room is visible. Spectrum (b) has a coincidence condition with scattered particles, but no Doppler correction is applied. Spectrum (c) is Doppler corrected assuming that the silicon detector was aligned with the beam axis. The misalignment of the silicon detector was included in the Doppler correction for spectrum (d), resulting in the final spectrum of Fig. 7 (for the full energy range and on a logarithmic scale). All spectra are shown without any background subtraction. data. The transitional matrix elements are related to the reduced E2 transition probabilities as:

$$
B\left(E 2 ; I_{1} \rightarrow I_{2}\right)=\frac{\left|\left\langle I_{2}\|\mathcal{M}(E 2)\| I_{1}\right\rangle\right|^{2}}{2 I_{1}+1} .
$$

The transitional quadrupole moment in the intrinsic reference frame can be expressed in the rotational model as:

$$
e Q_{0}^{t}=\sqrt{\frac{16 \pi}{5}} \frac{1}{\sqrt{2 I_{1}+1}} \frac{\left\langle I_{2}\|\mathcal{M}(E 2)\| I_{1}\right\rangle}{\left\langle I_{1} K_{1} 20 \mid I_{2} K_{2}\right\rangle} .
$$

Note that one has to make assumptions about the $K$ quantum numbers of the initial and final states to express the matrix element as a transitional quadrupole moment, whereas the $B(E 2)$ value is model independent. The signs of the transitional matrix elements are relative and give no additional information about the shape.

The diagonal matrix element is a direct measure of the spectroscopic quadrupole moment $Q_{s}(I)$ of the state in the laboratory frame and, using the sign convention of Alder and Winther [9], can be written as:

$$
e Q_{s}=\sqrt{\frac{16 \pi}{5}} \frac{\langle I I 20 \mid I I\rangle}{\sqrt{2 I+1}}\langle I\|\mathcal{M}(E 2)\| I\rangle .
$$

The diagonal matrix elements are related to the static quadrupole moments of the nuclear state in the intrinsic frame and therefore to the charge distribution of the nucleus in that state:

$$
e Q_{0}^{s}=\sqrt{\frac{16 \pi}{5}} \frac{1}{\sqrt{2 I+1}} \frac{\langle I\|\mathcal{M}(E 2)\| I\rangle}{\langle I K 20 \mid I K\rangle} .
$$

In order to extract the static quadrupole moment from the diagonal matrix element an assumption on the $K$ value of the state is again needed. The sign of the diagonal matrix element is related to the type of quadrupole deformation: for $K=0$ a negative value corresponds to a prolate and a positive value to an oblate deformation in the intrinsic frame of the nucleus.

\section{B. Matrix elements for ${ }^{76} \mathrm{Kr}$}

In the case of ${ }^{76} \mathrm{Kr}$ the $\chi^{2}$ minimization was performed with $33 E 2$ and $5 M 1$ matrix elements. Branching ratios and $E 2 / M 1$ mixing ratios from previous work were used as additional data for the fit; they are summarized in Table III. The lifetimes of all states that enter into the Coulomb excitation 
TABLE III. Relative intensities $I_{\gamma}[13,17]$ and mixing parameters $\delta[13]$ for mixed $E 2 / M 1$ transitions in ${ }^{76} \mathrm{Kr}$.

\begin{tabular}{rrrlr}
\hline \hline$I_{i}^{\pi}$ & $I_{f}^{\pi}$ & $E_{\gamma}(\mathrm{keV})$ & \multicolumn{1}{c}{$I_{\gamma}$} & $\delta(E 2 / M 1)$ \\
\hline $2_{2}^{+}$ & $2_{1}^{+}$ & 797 & 1.0 & $0.2(1)$ \\
$2_{2}^{+}$ & $0_{1}^{+}$ & 1221 & $0.69(4)$ & \\
$2_{3}^{+}$ & $0_{2}^{+}$ & 918 & 1.0 & \\
$2_{3}^{+}$ & $0_{1}^{+}$ & 1688 & $0.288(10)$ & \\
$2_{3}^{+}$ & $2_{1}^{+}$ & 1264 & $0.212(7)$ & \\
$2_{3}^{+}$ & $4_{1}^{+}$ & 653 & $0.092(3)$ & \\
$2_{3}^{+}$ & $2_{2}^{+}$ & 467 & $0.046(16)$ & \\
$4_{2}^{+}$ & $4_{1}^{+}$ & 923 & 1.0 & $-0.84(5)$ \\
$4_{2}^{+}$ & $2_{2}^{+}$ & 736 & $0.56(15)$ & \\
$4_{2}^{+}$ & $2_{1}^{+}$ & 1533 & $0.28(8)$ & \\
\hline \hline
\end{tabular}

analysis except those of the $2_{3}^{+}$and the $6_{2}^{+}$states were experimentally known. They are summarized in Table IV. To test the consistency between the lifetime and Coulomb excitation measurements, the $\chi^{2}$ minimization was first performed without using the lifetimes as additional input data. The resulting lifetimes of the states in the ground-state band agree with the measured values from Ref. $[18,19]$ within the experimental uncertainties. The analysis for ${ }^{74} \mathrm{Kr}$, however, revealed significant discrepancies between the lifetimes extracted from the Coulomb excitation data and those found in the literature, as will be discussed in the next chapter. These inconsistencies prompted a new lifetime measurement for several states in both ${ }^{74} \mathrm{Kr}$ and ${ }^{76} \mathrm{Kr}$ with improved accuracy [20], the results of which are also presented in Table IV. The lifetime found for the $2_{1}^{+}$state is longer than that of Ref. [18], while that for the $4_{1}^{+}$state is shorter. The results from the Coulomb excitation experiment are in between the values of the two measurements. The measured lifetimes were then used as additional input data

TABLE IV. Lifetimes of the relevant states in ${ }^{76} \mathrm{Kr}$. The values from various measurements are compared to a recent recoil-distance lifetime measurement [20] and to the results from the present Coulomb excitation experiment with and without the independently measured lifetimes as additional input data for the GOSIA fit of the matrix elements.

\begin{tabular}{cccccc}
\hline \hline$I^{\pi}$ & \multirow{2}{*}{$\tau(\mathrm{ps})$} & \multirow{2}{*}{ Ref. } & \multirow{2}{*}{$\tau(\mathrm{ps})[20]$} & \multicolumn{2}{c}{$\tau(\mathrm{ps})(\mathrm{GOSIA})$} \\
\cline { 5 - 6 } & & & & Free & Constr. \\
\hline $2_{1}^{+}$ & $36.0(10)$ & {$[18]$} & $41.5(8)$ & $38.0(22)$ & $41.2(6)$ \\
$4_{1}^{+}$ & $4.9(4)$ & {$[18]$} & $3.67(9)$ & $4.4(2)$ & $3.9(1)$ \\
$6_{1}^{+}$ & $0.86(10)$ & {$[19]$} & $0.97(29)$ & $0.82(5)$ & $0.76(6)$ \\
$8_{1}^{+}$ & $0.29(3)$ & {$[19]$} & & & $0.25(3)$ \\
$10_{1}^{+}$ & $0.14(2)$ & {$[19]$} & & & $0.15(3)$ \\
$0_{2}^{+}$ & $61.0(80)$ & {$[17]$} & & & $68.3(25)$ \\
$2_{2}^{+}$ & $1.4(2)$ & {$[13]$} & & & $1.6(1)$ \\
$4_{2}^{+}$ & $1.3(4)$ & {$[14]$} & & & $1.3(2)$ \\
$2_{3}^{+}$ & & & & & $0.47(5)$ \\
\hline \hline
\end{tabular}

in the GOSIA analysis, which enhanced the sensitivity to the diagonal matrix elements and the transitional matrix elements between higher-lying states. This enhanced sensitivity allowed extracting a lifetime also for the $2_{3}^{+}$state, which was previously unknown.

The transitional $E 2$ matrix elements found in the minimization with GOSIA are presented in Table V together with the transitional quadrupole moments and the $B(E 2)$ values. The diagonal matrix elements are given in Table VI together with the deduced static and spectroscopic quadrupole moments. The $M 1$ matrix elements for the mixed transitions between states of the same spin and parity are shown together with the corresponding $B(M 1)$ values in Table VII. All matrix elements are treated equally in fit; they are shown separately for clarity of the presentation. The results are compared to theoretical calculations, which will be discussed in Sec. V. Some of the transitional $E 2$ matrix elements between high-lying non-yrast states and the diagonal matrix elements for some of the higher-lying states could not be established within meaningful errors and are omitted in Tables V and VI. Nevertheless they entered into the $\chi^{2}$ minimization. The same is true for some of the $M 1$ matrix elements of the transitions between states of the same spin.

Starting values and signs of the matrix elements have been systematically changed in the initialization of the fit and also during the minimization procedure in order to avoid trapping in local $\chi^{2}$ minima. The signs of the transitional matrix elements are relative. Multiple combinations of signs can result in exactly the same population of the states. Positive signs were chosen for the transitional matrix elements in the ground-state band and other in-band transitions. The signs of all other transitional matrix elements are determined relative to these. The signs of the diagonal matrix elements are observables and cannot be chosen. Changing the sign of any of the diagonal matrix elements shown in Table VI results in a higher $\chi^{2}$ value of the fit. In addition, the relative phases of the transitional matrix elements between the different $0^{+}$and $2^{+}$states were investigated. Changing the sign of a closed loop of three matrix elements

$$
\begin{aligned}
& P_{3}\left(I_{1}^{\pi}, I_{2}^{\pi}, I_{3}^{\pi}\right) \\
& \quad=\left\langle I_{1}^{\pi}|| \mathcal{M}(E 2)|| I_{2}^{\pi}\right\rangle\left\langle I_{2}^{\pi}|| \mathcal{M}(E 2)|| I_{3}^{\pi}\right\rangle\left\langle I_{3}^{\pi}|| \mathcal{M}(E 2)|| I_{1}^{\pi}\right\rangle
\end{aligned}
$$

always resulted in a higher $\chi^{2}$ value, showing the sensitivity to the signs of the transitional matrix elements. However, the signs of the diagonal matrix elements remained the same when the sign of $P_{3}$ was inverted, showing the robustness of the fit for the diagonal matrix elements. As an example, the $\chi^{2}$ increases from 1.7 to 13 (after minimization) when changing the phase $P_{3}\left(0_{2}^{+}, 2_{1}^{+}, 2_{2}^{+}\right)$from positive to negative. In that case the diagonal matrix elements for the $2_{1}^{+}$and $2_{2}^{+}$states change from -0.9 to $-0.6 \mathrm{eb}$ and from -1.0 to $-0.5 \mathrm{eb}$, respectively.

To further illustrate the sensitivity of the fit to the diagonal matrix elements, the $\chi^{2}$ variation is shown as a function of the diagonal matrix elements for the $2_{1}^{+}, 4_{1}^{+}$and $2_{3}^{+}$states in Fig. 10. To find these $\chi^{2}$ values only the one diagonal matrix element in question was varied, and the $\gamma$-ray yields were calculated from the ensemble of all matrix elements. The $\chi^{2}$ functions for the different states are normalized to 
TABLE V. Reduced $E 2$ matrix elements, transitional quadrupole moments, and reduced transition probabilities for in-band and interband transitions in ${ }^{76} \mathrm{Kr}$. The $B(E 2)$ values are compared to theoretical results.

\begin{tabular}{|c|c|c|c|c|c|c|}
\hline$I_{1}^{\pi}$ & $I_{2}^{\pi}$ & $\left\langle I_{2}\|\mathcal{M}(E 2)\| I_{1}\right\rangle(e \mathrm{~b})$ & $Q_{0}^{t}(e \mathrm{~b})$ & $B\left(E 2 ; I_{1} \rightarrow I_{2}\right)\left(e^{2} \mathrm{~b}^{2}\right)$ & Skyrme [21] & Gogny \\
\hline $2_{1}^{+}$ & $0_{1}^{+}$ & $0.849_{-0.006}^{+0.006}$ & $2.69_{-0.02}^{+0.02}$ & $0.144_{-0.002}^{+0.002}$ & 0.202 & 0.117 \\
\hline $4_{1}^{+}$ & $2_{1}^{+}$ & $1.49_{-0.01}^{+0.01}$ & $2.94_{-0.03}^{+0.03}$ & $0.247_{-0.006}^{+0.006}$ & 0.281 & 0.234 \\
\hline $6_{1}^{+}$ & $4_{1}^{+}$ & $1.90_{-0.03}^{+0.11}$ & $2.98_{-0.06}^{+0.17}$ & $0.28_{-0.01}^{+0.03}$ & 0.39 & 0.30 \\
\hline $8_{1}^{+}$ & $6_{1}^{+}$ & $2.25_{-0.10}^{+0.16}$ & $3.02_{-0.14}^{+0.22}$ & $0.30_{-0.03}^{+0.05}$ & 0.44 & \\
\hline $10_{1}^{+}$ & $8_{1}^{+}$ & $2.19_{-0.14}^{+0.22}$ & $2.60_{-0.15}^{+0.26}$ & $0.23_{-0.03}^{+0.05}$ & & \\
\hline $2_{3}^{+}$ & $0_{2}^{+}$ & $0.87_{-0.02}^{+0.04}$ & $2.77_{-0.07}^{+0.12}$ & $0.15_{-0.01}^{+0.01}$ & 0.04 & 0.08 \\
\hline $4_{2}^{+}$ & $2_{2}^{+}$ & $0.89_{-0.13}^{+0.10}$ & $1.77_{-0.26}^{+0.20}$ & $0.09_{-0.03}^{+0.02}$ & & 0.11 \\
\hline $2_{3}^{+}$ & $0_{1}^{+}$ & $0.121_{-0.005}^{+0.004}$ & & $0.0029_{-0.0002}^{+0.0002}$ & 0.0033 & 0.0002 \\
\hline $2_{2}^{+}$ & $0_{1}^{+}$ & $0.183_{-0.006}^{+0.008}$ & & $0.0067_{-0.0004}^{+0.0005}$ & & 0.0010 \\
\hline $0_{2}^{+}$ & $2_{1}^{+}$ & $-0.490_{-0.008}^{+0.011}$ & & $0.241_{-0.009}^{+0.011}$ & 0.0001 & 0.234 \\
\hline $2_{3}^{+}$ & $2_{1}^{+}$ & $-0.200_{-0.008}^{+0.009}$ & & $0.0080_{-0.0007}^{+0.0007}$ & 0.0829 & 0.00006 \\
\hline $2_{2}^{+}$ & $2_{1}^{+}$ & $-0.09_{-0.04}^{+0.04}$ & & $0.002_{-0.002}^{+0.002}$ & & 0.148 \\
\hline $4_{2}^{+}$ & $2_{1}^{+}$ & $0.09_{-0.19}^{+0.01}$ & & $0.0010_{-0.0010}^{+0.0003}$ & & 0.0015 \\
\hline $2_{3}^{+}$ & $4_{1}^{+}$ & $0.52_{-0.05}^{+0.05}$ & & $0.055_{-0.010}^{+0.012}$ & 0.095 & 0.049 \\
\hline $2_{2}^{+}$ & $4_{1}^{+}$ & $-0.62_{-0.05}^{+0.04}$ & & $0.079_{-0.014}^{+0.014}$ & & 0.023 \\
\hline $4_{2}^{+}$ & $4_{1}^{+}$ & $0.43_{-0.03}^{+0.03}$ & & $0.021_{-0.003}^{+0.003}$ & & 0.076 \\
\hline $2_{2}^{+}$ & $0_{2}^{+}$ & $1.22_{-0.04}^{+0.08}$ & & $0.30_{-0.02}^{+0.04}$ & & 0.025 \\
\hline $2_{3}^{+}$ & $2_{2}^{+}$ & $0.81_{-0.24}^{+0.10}$ & & $0.13_{-0.07}^{+0.03}$ & & 0.04 \\
\hline
\end{tabular}

the same value in order to allow a quantitative comparison of their behavior. The $\chi^{2}$ curves resulting from the variation of the diagonal matrix elements for the $2_{1}^{+}$and $4_{1}^{+}$states are very narrow, showing that the fit is very sensitive to these matrix elements, which have consequently a relatively small uncertainty. The curve for the $2_{3}^{+}$state is much wider, so that this matrix element is less well defined and the error is larger. It should be noted, however, that this one-dimensional variation of the matrix elements serves only the purpose of illustrating the sensitivity of the fit. To find the values and errors of the matrix elements, all matrix elements are varied in a multidimensional fit including a full error analysis of the correlated parameters [22].

Both the angular distribution of the $\gamma$ rays and the deorientation of the nuclear alignment have to be taken into account in the $\chi^{2}$ minimization of the $\gamma$-ray yields. The deorientation is due to the interaction between the recoiling nucleus and fluctuating hyperfine fields created by atomic electrons. The effect attenuates the angular distribution of the $\gamma$ rays. The complex deorientation effect is treated in a simplifying phenomenological two-state deorientation model $[23,24]$. The most important parameters in this model are the spin and lifetime of the state, as well as its gyromagnetic factor. While the lifetimes of the states are either taken from a complementary measurement or come directly out of the GOSIA fit, the $g$ factors are mostly unknown. In these cases the $g$-factor values from the general approximation $g=Z / A$ can be used, even though there can be significant deviations from that value depending on the structure of the state. The $g$ factor of the $2_{1}^{+}$state in ${ }^{76} \mathrm{Kr}$ was recently measured to be $g=+0.37(11)$ [25]. The influence of the $g$ factor and the deorientation effect on the matrix elements was investigated

TABLE VI. Diagonal matrix elements, intrinsic, and spectroscopic quadrupole moments for ${ }^{76} \mathrm{Kr}$. The spectroscopic quadrupole moments are compared to theoretical values.

\begin{tabular}{crrrrr}
\hline \hline$I^{\pi}$ & $\langle I\|\mathcal{M}(E 2)\| I\rangle(e \mathrm{~b})$ & $Q_{0}^{s}(e \mathrm{~b})$ & $Q_{s}(e \mathrm{~b})$ & Skyrme [21] & Gogny \\
\hline $2_{1}^{+}$ & $-0.9_{-0.3}^{+0.3}$ & $2.5_{-0.8}^{+0.8}$ & $-0.7_{-0.2}^{+0.2}$ & -0.78 & -0.50 \\
$4_{1}^{+}$ & $-2.3_{-0.4}^{+0.4}$ & $4.7_{-0.8}^{+0.8}$ & $-1.7_{-0.3}^{+0.3}$ & -1.25 & -0.85 \\
$6_{1}^{+}$ & $-2.9_{-0.4}^{+0.4}$ & $5.1_{-0.7}^{+0.7}$ & $-2.0_{-0.3}^{+0.3}$ & -1.44 & -1.01 \\
$2_{3}^{+}$ & $1.3_{-0.5}^{+0.5}$ & $-3.4_{-1.3}^{+1.3}$ & $1.0_{-0.4}^{+0.4}$ & 0.25 & 0.04 \\
$2_{2}^{+}$ & $-1.0_{-0.5}^{+0.5}$ & $-2.6_{-1.3}^{+1.3 \mathrm{a}}$ & $-0.7_{-0.3}^{+0.3}$ & & 0.26 \\
\hline \hline
\end{tabular}

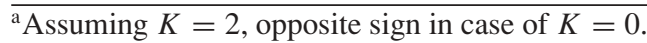


TABLE VII. Reduced $M 1$ matrix elements between states of the same spin and parity in ${ }^{76} \mathrm{Kr}$ and the corresponding $B(M 1)$ values.

\begin{tabular}{cccc}
\hline \hline$I_{1}^{\pi}$ & $I_{2}^{\pi}$ & $\left\langle I_{2}\|\mathcal{M}(M 1)\| I_{1}\right\rangle\left(\mu_{N}\right)$ & $B\left(M 1 ; I_{1} \rightarrow I_{2}\right)\left(\mu_{N}^{2}\right)$ \\
\hline $2_{2}^{+}$ & $2_{1}^{+}$ & $-0.42(1)$ & $0.035(2)$ \\
$4_{2}^{+}$ & $4_{1}^{+}$ & $-0.39(3)$ & $0.017(3)$ \\
\hline \hline
\end{tabular}

by comparing the results obtained using the measured $g$ factor with those using $g=Z / A=0.47$, or ignoring the deorientation effect entirely. No difference was found for the transitional matrix elements when including the deorientation effect or not, and when using $Z / A$ or the measured value. The diagonal matrix element of the $2_{1}^{+}$state differed by $8 \%$ when the deorientation was not included in the calculation. The difference between using $g=0.37$ and $g=0.47$, however, was negligible. Consequently, the approximation $g=Z / A$ was used to model the deorientation effect for the higher-lying states in ${ }^{76} \mathrm{Kr}$ and for all states in ${ }^{74} \mathrm{Kr}$.

The fact that the $2_{3}^{+} \rightarrow 0_{2}^{+}$transition with $918 \mathrm{keV}$ cannot be fully resolved from the $4_{2}^{+} \rightarrow 4_{1}^{+}$transition with $923 \mathrm{keV}$ requires particular attention for the evaluation of the respective matrix elements. It is possible to include such doublets in the GOSIA analysis by only fixing the sum of both $\gamma$-ray intensities rather than using the individual ones. This results necessarily in less accurate values for the matrix elements. The transitional matrix elements that are found without any further assumptions about the doublet show that the transition strength for the $2_{3}^{+} \rightarrow 0_{2}^{+}$transition is much higher than for the $4_{2}^{+} \rightarrow 4_{1}^{+}$transition. In addition to that, the population of the $4_{2}^{+}$state is expected to be much weaker than for the $2_{3}^{+}$ state, because it requires at least a two-step excitation, while the rather collective $2_{3}^{+}$state at a similar excitation energy can be reached in one step. If the $4_{2}^{+}$state were populated, not only its decay to the $4_{1}^{+}$state, but also the $736 \mathrm{keV}$ transition to the $2_{2}^{+}$state should be observed. This branching ratio was reported to be 0.56 [13] and 0.82 [19]. Because there is no convincing evidence in the spectra for a $736 \mathrm{keV}$ transition

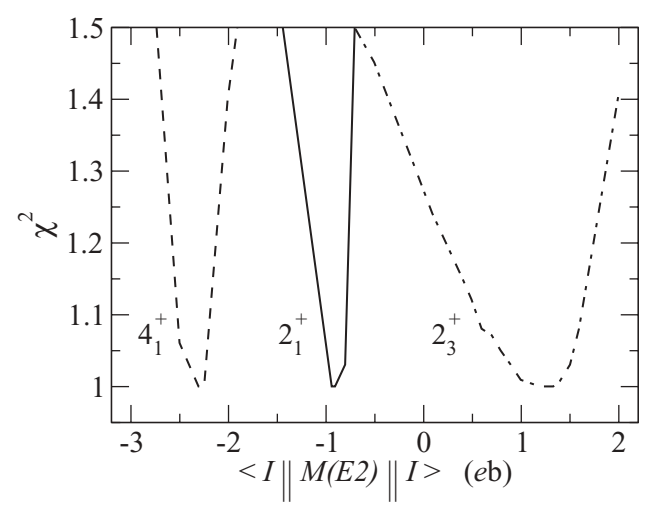

FIG. 10. Normalized $\chi^{2}$ curves as a function of the diagonal matrix elements of the $2_{1}^{+}, 4_{1}^{+}$, and $2_{3}^{+}$states in ${ }^{76} \mathrm{Kr}$. Only the one matrix element in question was varied in the calculation of the $\gamma$-ray yields to illustrate the sensitivity of the $\chi^{2}$ fit to this matrix element. (see Figs. 2 and 5) it can be assumed that the $4_{2}^{+}$state is only weakly populated, if at all, and that the contribution of the $4_{2}^{+} \rightarrow 4_{1}^{+}$transition to the peak observed at $920 \mathrm{keV}$ can be neglected. This assumption improves the accuracy for both the transitional matrix elements of the respective transitions and for the diagonal matrix element of the $2_{3}^{+}$state. Following this argumentation, no decay from the $4_{2}^{+}$state is observed, and consequently the diagonal matrix element for this state cannot be determined.

The matrix elements of Table $\mathrm{V}$ are grouped into in-band transitions in the upper part of the table and interband transitions in the lower part. The in-band transitions comprise all transitions of the ground-state band and the $2_{3}^{+} \rightarrow 0_{2}^{+}$and $4_{2}^{+} \rightarrow 2_{2}^{+}$transitions (see Fig. 3). All in-band transitions have large matrix elements, i.e., they are collective, as expected for well-deformed rotational bands. The deduced $B(E 2)$ values are fully compatible with those extracted from the measured lifetimes $[19,20]$. The drop of the transitional quadrupole moments $Q_{0}^{t}$ in the lower part of the ground-state band has been interpreted as a sign for mixing of prolate and oblate configurations in the low-spin states [20]. This interpretation is further supported by the large matrix elements for some of the interband transitions. The fact that the $0_{2}^{+}$state is strongly linked to all three observed $2^{+}$states, however, indicates that a grouping of the states into rotational bands is not straightforward and that the situation might be more complex than the description with two rotational bands built on strongly deformed prolate and oblate states together with a quasi-gamma band. The transitional matrix elements allow to determine previously unknown lifetimes, for example, for the $2_{3}^{+}$state, or to improve the precision of previously known lifetimes, as is summarized in Table IV.

The diagonal matrix elements for the three lowest states of the ground-state band and for the two excited $2^{+}$states are given in Table VI together with the deduced static and spectroscopic quadrupole moments. The negative sign of the diagonal matrix elements for the states in the ground-state band proves their prolate shape. The absolute size of the quadrupole moments decreases toward the bottom of the band. This shows that the deformation for the $2_{1}^{+}$state is indeed smaller than for the states above. In addition, the values of the static and transitional quadrupole moments for this state are rather similar, as expected for a rotational nucleus. Very large static quadrupole moments are found for the higher-spin states in the ground-state band, which differ from the transitional quadrupole moments. This might be due to a possible coupling to other unknown states, which could not be taken into account in the analysis.

The diagonal matrix element of the $2_{3}^{+}$state has a relatively large uncertainty, mainly because no transition above this state was observed. Nevertheless there is no doubt about the positive sign of the matrix element, which is consistent with the assumption of an oblate-deformed $K=0$ band. The large $E 2$ matrix element to the $0_{2}^{+}$state further supports the assumption of a rotational character of the state, so that the association of the $2_{3}^{+}$state with an oblate shape seems well justified.

The negative sign of the matrix element for the $2_{2}^{+}$state is more difficult to understand. In case of a quasi-gamma band (with $K=2$ ), the quadrupole moment in the body-fixed 
TABLE VIII. Branching ratios for several transitions in ${ }^{74} \mathrm{Kr}$ measured after $\beta$ decay of ${ }^{74} \mathrm{Rb}$ [15].

\begin{tabular}{llrl}
\hline \hline$I_{i}^{\pi}$ & $I_{f}^{\pi}$ & $E_{\gamma}(\mathrm{keV})$ & \multicolumn{1}{c}{$I_{\gamma}$} \\
\hline $2_{2}^{+}$ & $0_{1}^{+}$ & 1202 & 1.0 \\
$2_{2}^{+}$ & $2_{1}^{+}$ & 746 & $0.73(58)$ \\
$2_{2}^{+}$ & $0_{2}^{+}$ & 694 & $0.30(35)$ \\
$2_{3}^{+}$ & $0_{2}^{+}$ & 1233 & 1.0 \\
$2_{3}^{+}$ & $2_{1}^{+}$ & 1285 & $0.31(21)$ \\
$0_{2}^{+}$ & $0_{1}^{+}$ & $508^{\mathrm{a}}$ & 1.0 \\
$0_{2}^{+}$ & $2_{1}^{+}$ & 52 & $1.50(36)$ \\
$0_{2}^{+}$ & $2_{1}^{+}$ & 52 & $1.2(5)^{\mathrm{b}}$ \\
\hline \hline
\end{tabular}

${ }^{\mathrm{a}} \mathrm{E} 0$ transition proceeding via internal conversion.

${ }^{\mathrm{b}}$ Branching ratio from Ref. [7].

frame $Q_{0}^{s}$ becomes negative, indicating an oblate shape, in contradiction to the assumption of a gamma vibration based on the prolate ground state. Indeed, the coupling of the $2_{2}^{+}$state to the $0_{2}^{+}$state is much stronger than that to the ground state. If one assumes $K=0$ for the $2_{2}^{+}$state, the quadrupole moment $Q_{0}^{s}$ becomes positive, indicating a prolate shape, in which case the interpretation of a quasi-gamma band is also excluded, besides the fact that there should be a third $0^{+}$state for which there is no evidence. As was argued before, the strong coupling between all three bands and in particular between the $0_{2}^{+}$state and all three $2^{+}$states blurs a clear separation and grouping into band structures, as will be discussed in more detail in section V.

\section{Matrix elements for ${ }^{74} \mathrm{Kr}$}

The $\chi^{2}$ minimization of the $\gamma$-ray yields for ${ }^{74} \mathrm{Kr}$ was performed with $31 E 2$ and $5 M 1$ matrix elements connecting the known states as shown in Fig. 3. As in the case of ${ }^{76} \mathrm{Kr}$, experimentally known branching ratios and lifetimes were used as additional input data in the fitting procedure. The branching ratios that were recently measured after $\beta$ decay of ${ }^{74} \mathrm{Rb}[15]$ are summarized in Table VIII. The decay of the $\mathrm{O}_{2}^{+}$state represents a special case because the $E 0$ branch to the ground state proceeds exclusively via conversion electrons and the $E 2$ branch to the $2_{1}^{+}$state has very low energy and is consequently also highly converted. Even though the Coulomb excitation experiment was not sensitive to conversion electrons, the decay of this state with its branching ratio and partial lifetimes [7] can nevertheless be used to constrain the fit. No mixing ratios are known in ${ }^{74} \mathrm{Kr}$.

Lifetimes in ${ }^{74} \mathrm{Kr}$ have been measured for the $2_{1}^{+}$and $4_{1}^{+}$ states using the recoil-distance method [26,27], and using the Doppler-shift attenuation method for the higher-lying states of the ground-state band $[26,28,29]$. The lifetime of the isomeric $\mathrm{O}_{2}^{+}$state was established in a conversion-electron measurement [7]. The lifetime values are summarized in Table IX.

Before using the literature values for the lifetimes as complementary input in the fitting procedure, their consistency with the Coulomb excitation data was examined. The results, also given in Table IX, show a strong deviation from the values reported in Ref. [26,27]. Especially the lifetime of the $4_{1}^{+}$state is found to be significantly shorter than previously reported.
TABLE IX. Lifetimes of states in ${ }^{74} \mathrm{Kr}$. The values from various measurements are compared to a new recoil-distance lifetime measurement [20] and to the results from the Coulomb excitation experiment with and without the lifetimes from Ref. [20] as complementary input data.

\begin{tabular}{lllllc}
\hline \hline$I^{\pi}$ & \multirow{2}{*}{$\tau(\mathrm{ps})$} & \multirow{2}{*}{ Ref. $\tau(\mathrm{ps})[20]$} & \multicolumn{2}{c}{$\tau(\mathrm{ps})(\mathrm{GOSIA})$} \\
\cline { 3 - 6 } & & & & Free & Constr. \\
\hline $2_{1}^{+}$ & $23.5(20)$ & {$[26]$} & $33.8(6)$ & $29.6(2.1)$ & $33.8(6)$ \\
$4_{1}^{+}$ & $13.2(7)$ & {$[27]$} & $5.2(2)$ & $5.9(5)$ & $5.3(2)$ \\
$6_{1}^{+}$ & $1.08(14)$ & {$[29]$} & $1.09(23)$ & $1.4(5)$ & $1.01(9)$ \\
$8_{1}^{+}$ & $0.35(5)$ & {$[29]$} & & & $0.32(6)$ \\
$10_{1}^{+}$ & $0.16(3)$ & {$[29]$} & & & $0.16(3)$ \\
$0_{2}^{+}$ & $33.8(50) \cdot 10^{3 \mathrm{a}}$ & {$[7]$} & & & $36.2(43) \cdot 10^{3 \mathrm{a}}$ \\
$2_{2}^{+}$ & & & & & $2.0(2)$ \\
$0_{3}^{+}$ & & & & & $0.09(2)^{\mathrm{a}}$ \\
\hline \hline
\end{tabular}

a Partial lifetime of E2 branch to the $2_{1}^{+}$state.

However, using the lifetimes of the $2_{1}^{+}$and $4_{1}^{+}$states in the GOSIA fit to enhance the sensitivity to the diagonal matrix elements is even more important in ${ }^{74} \mathrm{Kr}$ than it was in ${ }^{76} \mathrm{Kr}$, as the level of statistics is more limited.

The inconsistency between the earlier lifetime measurements and the Coulomb excitation data can be further investigated by comparing the experimental $\gamma$-ray intensities as a function of scattering angle with those calculated from the matrix elements obtained using the lifetimes. This is shown in Fig. 11 for the $2_{1}^{+} \rightarrow 0_{1}^{+}$and $4_{1}^{+} \rightarrow 2_{1}^{+}$transitions. The data points represent the observed $\gamma$-ray intensities normalized by the number of scattered projectiles for several angular ranges. The uncertainties are large for small angles because of direct beam hitting the innermost detector rings. The dashed lines show the $\gamma$-ray yields calculated from the full set of matrix elements obtained in a fit that was constrained by the lifetimes reported by Tabor et al. for the $2_{1}^{+}$[26] and by

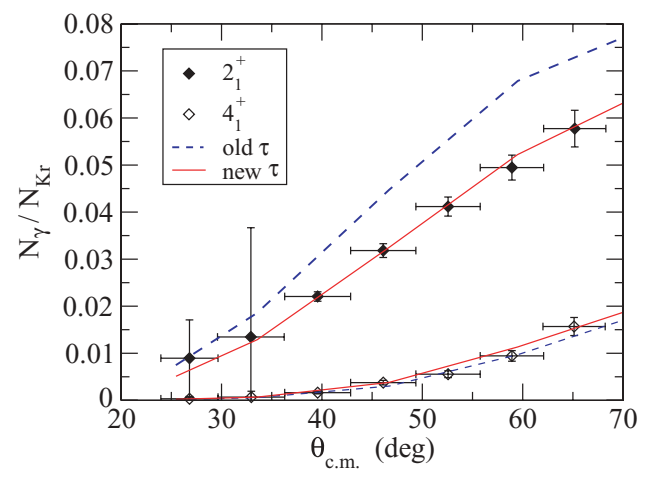

FIG. 11. (Color online) Gamma-ray intensities normalized by the number of scattered projectiles as a function of the center-of-mass scattering angle for the $2_{1}^{+}$and $4_{1}^{+}$states in ${ }^{74} \mathrm{Kr}$. The dashed lines show the $\gamma$-ray yields calculated from the matrix elements that were obtained using the lifetimes of the $2_{1}^{+}[26]$ and $4_{1}^{+}$states [27] in the GOSIA fit. Using the lifetimes from Ref. [20] instead results in the full lines. 
TABLE X. Reduced E2 matrix elements, transitional quadrupole moments, and reduced transition probabilities for in-band and interband transitions in ${ }^{74} \mathrm{Kr}$. The $B(E 2)$ values are compared to theoretical results.

\begin{tabular}{ccccccc}
\hline \hline$I_{1}^{\pi}$ & $I_{2}^{\pi}$ & $\left\langle I_{2}\|\mathcal{M}(E 2)\| I_{1}\right\rangle(e \mathrm{~b})$ & $Q_{0}^{t}(e \mathrm{~b})$ & $B\left(E 2 ; I_{1} \rightarrow I_{2}\right)\left(e^{2} \mathrm{~b}^{2}\right)$ & Skyrme [21] & Gogny \\
\hline $2_{1}^{+}$ & $0_{1}^{+}$ & $0.782_{-0.007}^{+0.007}$ & $2.48_{-0.02}^{+0.02}$ & $0.122_{-0.002}^{+0.002}$ & 0.188 & 0.104 \\
$4_{1}^{+}$ & $2_{1}^{+}$ & $1.60_{-0.03}^{+0.03}$ & $3.16_{-0.05}^{+0.05}$ & $0.285_{-0.009}^{+0.010}$ & 0.340 & 0.248 \\
$6_{1}^{+}$ & $4_{1}^{+}$ & $1.98_{-0.09}^{+0.10}$ & $3.10_{-0.13}^{+0.15}$ & $0.30_{-0.03}^{+0.03}$ & 0.41 & 0.34 \\
$8_{1}^{+}$ & $6_{1}^{+}$ & $2.25_{-0.16}^{+0.23}$ & $3.02_{-0.21}^{+0.31}$ & $0.30_{-0.04}^{+0.07}$ & 0.45 & 0.48 \\
$10_{1}^{+}$ & $8_{1}^{+}$ & $2.35_{-0.18}^{+0.29}$ & $2.79_{-0.22}^{+0.35}$ & $0.26_{-0.04}^{+0.07}$ & 0.027 & 0.045 \\
$2_{2}^{+}$ & $0_{2}^{+}$ & $-0.48_{-0.04}^{+0.03}$ & $-1.51_{-0.11}^{+0.10}$ & $0.045_{-0.007}^{+0.007}$ & 0.120 & 0.118 \\
$4_{2}^{+}$ & $2_{2}^{+}$ & $-0.55_{-0.08}^{+0.16}$ & $-1.10_{-0.15}^{+0.33}$ & $0.035_{-0.011}^{+0.020}$ & 0.120 .014 & 0.0014 \\
$2_{2}^{+}$ & $0_{1}^{+}$ & $-0.199_{-0.011}^{+0.018}$ & & $0.0079_{-0.0009}^{+0.0009}$ & 0.0147 & 0.00002 \\
$2_{3}^{+}$ & $0_{1}^{+}$ & $-0.172_{-0.014}^{+0.021}$ & & $0.0059_{-0.0010}^{+0.0010}$ & & 0.36 \\
$0_{2}^{+}$ & $2_{1}^{+}$ & $0.68_{-0.03}^{+0.04}$ & & $0.47_{-0.05}^{+0.05}$ & 0.18 & 0.172 \\
$2_{2}^{+}$ & $2_{1}^{+}$ & $0.49_{-0.04}^{+0.04}$ & & $0.047_{-0.008}^{+0.007}$ & 0.055 & 0.0009 \\
$0_{3}^{+}$ & $2_{1}^{+}$ & $0.59_{-0.05}^{+0.07}$ & & $0.35_{-0.06}^{+0.09}$ & & 0.066 \\
$2_{2}^{+}$ & $4_{1}^{+}$ & $0.47_{-0.20}^{+0.28}$ & & $0.045_{-0.029}^{+0.070}$ & 0.043 & 0.06 \\
$2_{3}^{+}$ & $0_{2}^{+}$ & $0.68_{-0.27}^{+0.27}$ & & $0.09_{-0.06}^{+0.09}$ & & \\
\hline \hline
\end{tabular}

Roth et al. for the $4_{1}^{+}$state [27]. The range of scattering angles for this comparison was limited to small values $\theta_{\mathrm{cm}}<70^{\circ}$, where higher-order effects and in particular the influence of the diagonal matrix elements are expected to be small, so that this comparison of $\gamma$-ray yields represents a relatively clean test of the $B(E 2)$ strengths and of the lifetimes of the respective states. The fact that the $\gamma$-ray yields cannot be reproduced simultaneously for both transitions shows that the Coulomb excitation data are not compatible with the lifetimes reported in Ref. [26,27].

This inconsistency, which makes the determination of the diagonal matrix elements difficult, prompted a new recoildistance lifetime experiment with much improved precision [20]. The new measurement found slightly longer and shorter lifetimes for the $2_{1}^{+}$and $4_{1}^{+}$states, respectively, than expected from the Coulomb excitation data, but especially the result for the $4_{1}^{+}$state is deviating significantly from the value reported by Roth et al. [27]. Possible reasons for this are discussed in Ref. [20]. The new results are compared to the earlier measurements and to the results from the Coulomb excitation data in Table IX. When the lifetimes of the new measurement are used in the GOSIA fit, the inconsistencies disappear. The $\gamma$-ray yields calculated from the re-evaluated matrix elements based on the new lifetime results are shown as full lines in Fig. 11. Even though the new lifetimes have small uncertainties and leave very little room for variations of the calculated $\gamma$-ray yields in the shown angular range, the agreement is excellent, which shows the full consistency between the recoil-distance and the Coulomb excitation measurements.

Using the new precise lifetimes in the GOSIA analysis enhances the sensitivity to the higher-order effects significantly, so that many more transitional matrix elements between higher-lying states and several diagonal matrix elements could be determined. The results for the transitional matrix elements are summarized in Table X; those for the diagonal matrix elements and the static quadrupole moments can be found in Table XI. The inconsistency with the previously reported lifetimes illustrates a general difficulty that can arise in Coulomb excitation experiments with weak radioactive beams of rare isotopes, where the statistics is too limited to determine all parameters in the Coulomb excitation analysis alone, and where at the same time the access to spectroscopic information with complementary methods is difficult.

TABLE XI. Diagonal matrix elements, intrinsic, and spectroscopic quadrupole moments for ${ }^{74} \mathrm{Kr}$. The spectroscopic quadrupole moments are compared to theoretical values.

\begin{tabular}{cccccc}
\hline \hline$I^{\pi}$ & $\langle I\|\mathcal{M}(E 2)\| I\rangle(e \mathrm{~b})$ & $Q_{0}^{s}(e \mathrm{~b})$ & $Q_{s}(e \mathrm{~b})$ & Skyrme [21] & Gogny \\
\hline $2_{1}^{+}$ & $-0.70_{-0.30}^{+0.33}$ & $1.85_{-0.79}^{+0.85}$ & $-0.53_{-0.23}^{+0.24}$ & -0.91 & -0.48 \\
$4_{1}^{+}$ & $-1.0_{-0.2}^{+0.6}$ & $2.1_{-0.4}^{+1.2}$ & $-0.8_{-0.2}^{+0.4}$ & -1.31 & -0.98 \\
$6_{1}^{+}$ & $-1.8_{-0.7}^{+0.5}$ & $3.1_{-1.3}^{+0.8}$ & $-1.3_{-0.5}^{+0.3}$ & -1.46 & \\
$2_{2}^{+}$ & $0.33_{-0.23}^{+0.28}$ & $\pm 0.86_{-0.60}^{+0.73 a}$ & $0.24_{-0.17}^{+0.21}$ & 0.43 & 0.09 \\
$2_{3}^{+}$ & $0.4_{-0.4}^{+1.1}$ & $\pm 1.1_{-1.0}^{+3.1 \mathrm{a}}$ & $0.3_{-0.3}^{+0.9}$ & & 0.37 \\
\hline \hline
\end{tabular}

${ }^{2}$ Sign depending on $K$ : negative for $K=0$ and positive for $K=2$. 


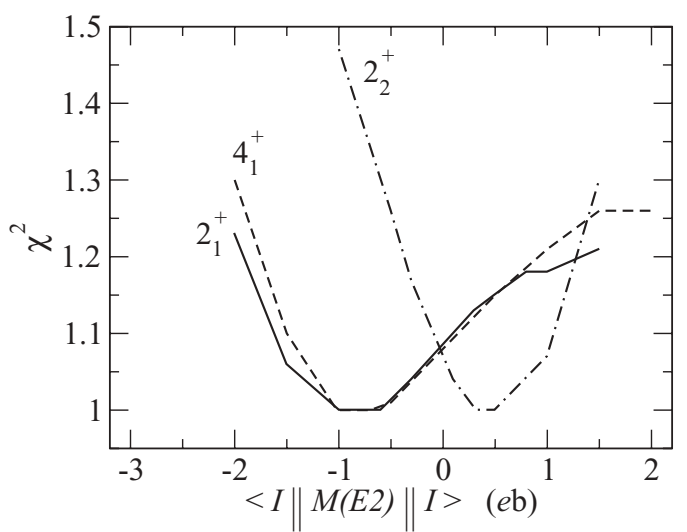

FIG. 12. Normalized $\chi^{2}$ curves as a function of the diagonal matrix elements of the $2_{1}^{+}, 4_{1}^{+}$, and $2_{2}^{+}$states in ${ }^{74} \mathrm{Kr}$. Only the one matrix element in question was varied in the calculation of the $\gamma$-ray yields to illustrate the sensitivity of the $\chi^{2}$ fit to this matrix element.

To illustrate the sensitivity of the Coulomb excitation analysis to the diagonal matrix elements, $\chi^{2}$ curves for the $2_{1}^{+}, 4_{1}^{+}$, and $2_{2}^{+}$states are shown in Fig. 12 as functions of the diagonal matrix elements of the respective states. The curves are normalized in exactly the same way as in Fig. 10 to allow a direct comparison. The $\chi^{2}$ minima are wider than in the ${ }^{76} \mathrm{Kr}$ case, which is mostly due to the lower level of statistics in the ${ }^{74} \mathrm{Kr}$ experiment. Again it should be noted that these curves cannot be used for an error analysis, as only one matrix element was varied at a time, whereas the uncertainties given in Tables X and XI are based on the simultaneous fit of the correlated matrix elements.

The high level of statistics for the transitions in the ground-state band allows extracting the $\gamma$-ray intensities for smaller angular bins than the four ranges that were used in the GOSIA analysis (see Table II). The intensities of the $4_{1}^{+} \rightarrow 2_{1}^{+}$and the $6_{1}^{+} \rightarrow 4_{1}^{+}$transitions are shown in the upper part of Fig. 13 as functions of the scattering angle. The intensities are normalized to the $2_{1}^{+} \rightarrow 0_{1}^{+}$transition to minimize the systematic errors from the efficiencies of the germanium and silicon detectors. The full lines correspond to the $\gamma$-ray intensities calculated from the complete set of matrix elements found in the $\chi^{2}$ minimization (see Tables $\mathrm{X}$ and XI). Even though the matrix elements were extracted from the $\gamma$-ray yields of only four angular ranges, the agreement with the more detailed analysis is remarkable and shows the consistency of the analysis. The dashed line results from inverting the sign of the diagonal matrix elements for the states of the ground-state band. The inversion of the signs leads to a disagreement especially for the large center-of-mass scattering angles, i.e. for small impact parameters and a close approach of projectile and target, while the small scattering angles are mostly sensitive to the first-order excitation process, i.e., to the $B(E 2)$ values, as was discussed in the context of Fig. 11. The difference between the yields calculated for different signs of the diagonal matrix elements illustrates the sensitivity of the measurement to the reorientation effect. Again, this cannot be used for a quantitative analysis, because only some selected matrix elements were arbitrarily changed, while both the transitional and the diagonal matrix elements of

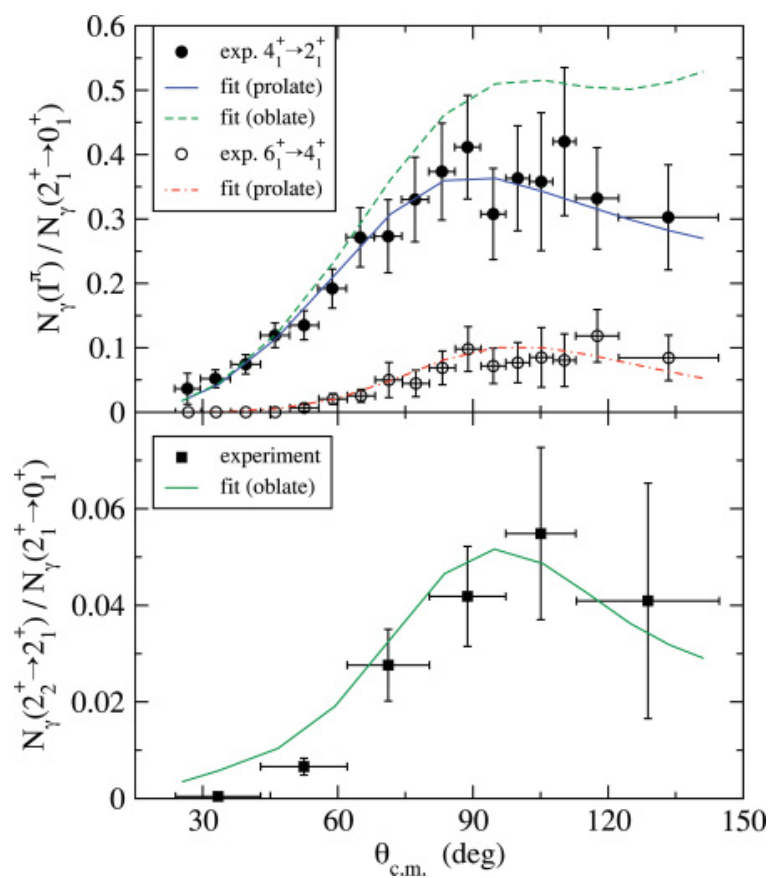

FIG. 13. (Color online) $\gamma$-ray yields as a function of scattering angle for the $4_{1}^{+} \rightarrow 2_{1}^{+}, 6_{1}^{+} \rightarrow 4_{1}^{+}$(top) and $2_{2}^{+} \rightarrow 2_{1}^{+}$(bottom) transitions in ${ }^{74} \mathrm{Kr}$ normalized to the $2_{1}^{+} \rightarrow 0_{1}^{+}$transition. The lines represent the corresponding yields based on the full set of matrix elements. The dashed line in the upper graph resulted from an inversion of the signs of the diagonal matrix elements for the states in the ground-state band.

the non-yrast states were kept unchanged. The second graph shows the equivalent plot for the $2_{2}^{+} \rightarrow 2_{1}^{+}$transition, also normalized to the $2_{1}^{+} \rightarrow 0_{1}^{+}$transition. Because the yield is smaller for this transition, the intensity has to be divided into fewer and wider angular bins. The agreement with the yield calculated from the matrix elements is yet another example for the consistency of the analysis.

The transitional matrix elements in Table $\mathrm{X}$ are again grouped into the in-band transitions (as illustrated in Fig. 3) in the top part of the table and interband transitions in the bottom part. Only those matrix elements are shown that could be established with reasonable uncertainties. The transitions within the ground-state band show a decrease in the lower part of the band similar to the ${ }^{76} \mathrm{Kr}$ case. This has also been interpreted as a sign for a mixing of the low-spin states and shape coexistence [20]. The transitions of the excited band are less collective than the transitions of the ground-state band. Many of the interband transitions presented in the lower part of Table $\mathrm{X}$ are surprisingly strong. In particular the matrix element for the $0_{2}^{+} \rightarrow 2_{1}^{+}$transition is almost as large as that of the $2_{1}^{+} \rightarrow 0_{1}^{+}$transition. This supports the interpretation of a strong mixing between prolate and oblate shapes for the two $0^{+}$states, which was proposed in Ref. [7] based on the $\rho^{2}(E 0)$ strength and the distortion of the rotational ground-state band. Lifetimes can be extracted for those states for which the matrix elements of the depopulating transitions could be sufficiently well determined, yielding the previously unknown lifetimes 
of the $2_{2}^{+}$and $0_{3}^{+}$transitions. The results are summarized in Table IX.

The negative sign of the diagonal matrix elements for the states in the ground-state band proves their prolate character. The quadrupole moments indicate a decrease of deformation toward the ground state. The role of the $2_{2}^{+}$and $2_{3}^{+}$states is not entirely clear. If one assumes that the $0_{2}^{+}, 2_{2}^{+}$, and $4_{2}^{+}$states form a rotational band with $K=0$, which seems justified by the large matrix elements connecting the states, the positive sign of the $\left\langle 2_{2}^{+}\|\mathcal{M}(E 2)\| 2_{2}^{+}\right\rangle$matrix element corresponds to oblate shape. However, there is also a strong coupling between the $2_{3}^{+}$and $0_{2}^{+}$states, so that the $2_{3}^{+}$state, which has also a positive diagonal matrix element, is another candidate for an oblate rotational state built on the $0_{2}^{+}$state. In the latter case the $2_{2}^{+}$and $4_{2}^{+}$states could be explained as members of a gamma-vibrational $K=2$ band, whereas in the former case the $2_{3}^{+}$state could be interpreted as a $K=2$ state. Similar as for ${ }^{76} \mathrm{Kr}$, the initial picture of two coexisting rotational bands with prolate and oblate shapes is clearly too simple, as oblate and prolate as well as $K=0$ and $K=2$ components seem to be strongly mixed in the wave functions of the various states.

\section{DISCUSSION}

The large number of experimental electromagnetic matrix elements for the low-spin states in ${ }^{74} \mathrm{Kr}$ and ${ }^{76} \mathrm{Kr}$ allows a detailed discussion of the shape coexistence phenomenon in these nuclei. In particular, negative values were found for the diagonal matrix elements of several states in the ground-state band of both isotopes, confirming the prolate shape of these states. Positive matrix elements were found for excited $2^{+}$ states which are strongly connected to the $0_{2}^{+}$states in both ${ }^{74} \mathrm{Kr}$ and ${ }^{76} \mathrm{Kr}$, consistent with an oblate character of these states. These results confirm the prolate-oblate shape coexistence scenario in both isotopes under study. However, a closer inspection of the matrix elements of Tables V, VI, X, and XI shows that the actual scenario is more complicated. In particular the influence of the third $2^{+}$state observed in both isotopes and usually interpreted as member of a quasi-gamma band needs further investigation.

The strength of the $2_{1}^{+} \rightarrow 0_{1}^{+}$transition is strongly reduced compared to the other transitions of the ground-state bands in both isotopes in accordance with the assumption of increased mixing toward the band heads. The decrease is stronger in ${ }^{74} \mathrm{Kr}$ than in ${ }^{76} \mathrm{Kr}$, pointing to a stronger mixing in ${ }^{74} \mathrm{Kr}$. The static quadrupole moments, which measure the shape of the nucleus in the respective state, show a similar behavior.

The results are discussed in the following first in the context of a simplifying phenomenological two-level mixing model, and then on the basis of more elaborated nuclear structure calculations. Model-independent shape parameters based on the quadrupole sum rule method will be deduced and discussed in the final section.

\section{A. Shape coexistence in a two-state mixing model}

The two-state mixing model is a simple and useful first approach for interpreting the properties of physical states based on the mixing of different intrinsic configurations. The model is based on the assumption of two regular rotational bands with collective in-band transitions, but no "cross talk" between the intrinsic states of the different bands. The mixing of states with the same spin-parity $I^{\pi}$ is described by a mixing angle $\theta_{I}$. The model is often used to deduce mixing properties from the energy displacement of the states with respect to an ideal rotor, as was done for example in Ref. [7]. It is now possible to test the validity of the model description for the transitional matrix elements. The observed physical states $\left|I_{1,2}\right\rangle$ can be written as a linear combination of the intrinsic pure prolate and oblate states $\left|I_{\mathrm{pr}}\right\rangle$ and $\left|I_{\mathrm{ob}}\right\rangle$, respectively,

$$
\begin{aligned}
& \left|I_{1}\right\rangle=+\cos \theta_{I}\left|I_{\mathrm{pr}}\right\rangle+\sin \theta_{I}\left|I_{\mathrm{ob}}\right\rangle, \\
& \left|I_{2}\right\rangle=-\sin \theta_{I}\left|I_{\mathrm{pr}}\right\rangle+\cos \theta_{I}\left|I_{\mathrm{ob}}\right\rangle .
\end{aligned}
$$

The condition of no "cross talk" between the bands means that for all states $I, J$ :

$$
\left\langle I_{\mathrm{ob}}\|\mathcal{M}(E 2)\| J_{\mathrm{pr}}\right\rangle=0 .
$$

The observed matrix elements between the different $2^{+}$and $0^{+}$states can then be expressed in terms of the "pure" matrix elements and the mixing angles:

$$
\begin{aligned}
M_{11}= & \left\langle 2_{1}\|\mathcal{M}(E 2)\| 0_{1}\right\rangle \\
= & \sin \theta_{0} \sin \theta_{2}\left\langle 2_{\mathrm{ob}}\|\mathcal{M}(E 2)\| 0_{\mathrm{ob}}\right\rangle \\
& +\cos \theta_{0} \cos \theta_{2}\left\langle 2_{\mathrm{pr}}\|\mathcal{M}(E 2)\| 0_{\mathrm{pr}}\right\rangle, \\
M_{12}= & \left\langle 2_{1}\|\mathcal{M}(E 2)\| 0_{2}\right\rangle \\
= & \cos \theta_{0} \sin \theta_{2}\left\langle 2_{\mathrm{ob}}\|\mathcal{M}(E 2)\| 0_{\mathrm{ob}}\right\rangle \\
& -\sin \theta_{0} \cos \theta_{2}\left\langle 2_{\mathrm{pr}}\|\mathcal{M}(E 2)\| 0_{\mathrm{pr}}\right\rangle, \\
M_{21}= & \left\langle 2_{2}\|\mathcal{M}(E 2)\| 0_{1}\right\rangle \\
= & \sin \theta_{0} \cos \theta_{2}\left\langle 2_{\mathrm{ob}}\|\mathcal{M}(E 2)\| 0_{\mathrm{ob}}\right\rangle \\
& -\cos \theta_{0} \sin \theta_{2}\left\langle 2_{\mathrm{pr}}\|\mathcal{M}(E 2)\| 0_{\mathrm{pr}}\right\rangle, \\
= & \left\langle 2_{2}\|\mathcal{M}(E 2)\| 0_{2}\right\rangle \\
= & \cos \theta_{0} \cos \theta_{2}\left\langle 2_{\mathrm{ob}}\|\mathcal{M}(E 2)\| 0_{\mathrm{ob}}\right\rangle \\
& +\sin \theta_{0} \sin \theta_{2}\left\langle 2_{\mathrm{pr}}\|\mathcal{M}(E 2)\| 0_{\mathrm{pr}}\right\rangle .
\end{aligned}
$$

Solving the system of equations yields the following expressions:

$$
\tan \theta_{0}=A \pm \sqrt{A^{2}+1}
$$

with

$$
\begin{aligned}
A & =\frac{M_{11}^{2}+M_{21}^{2}-M_{12}^{2}-M_{22}^{2}}{2\left(M_{11} M_{12}+M_{21} M_{22}\right)}, \\
\tan \theta_{2} & =\frac{M_{11} \tan \theta_{0}+M_{12}}{M_{21} \tan \theta_{0}+M_{22}}, \\
\left\langle 2_{\mathrm{pr}}\|\mathcal{M}(E 2)\| 0_{\mathrm{pr}}\right\rangle & =\frac{M_{11} \cos \theta_{2}-M_{21} \sin \theta_{2}}{\cos \theta_{0}}, \\
\left\langle 2_{\mathrm{ob}}\|\mathcal{M}(E 2)\| 0_{\mathrm{ob}}\right\rangle & =\frac{M_{21} \cos \theta_{2}+M_{11} \sin \theta_{2}}{\sin \theta_{0}} .
\end{aligned}
$$

The latter matrix elements can be used to calculate the intrinsic quadrupole moments $Q_{0}$ for the bands with pure prolate and 


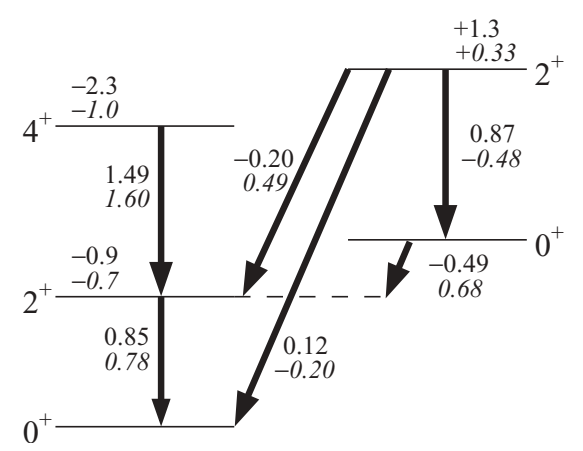

FIG. 14. Summary of the transitional and diagonal matrix elements for the relevant states in ${ }^{76} \mathrm{Kr}$ and ${ }^{74} \mathrm{Kr}$ (italic). Note that the excited $2^{+}$state corresponds to the $2_{2}^{+}$state in ${ }^{74} \mathrm{Kr}$ and to the $2_{3}^{+}$state in ${ }^{76} \mathrm{Kr}$.

oblate configuration, respectively:

$$
\begin{aligned}
Q_{0}^{\mathrm{pr}} & =\sqrt{\frac{16 \pi}{5}}\left\langle 2_{\mathrm{pr}}\|\mathcal{M}(E 2)\| 0_{\mathrm{pr}}\right\rangle, \\
Q_{0}^{\mathrm{ob}} & =\sqrt{\frac{16 \pi}{5}}\left\langle 2_{\mathrm{ob}}\|\mathcal{M}(E 2)\| 0_{\mathrm{ob}}\right\rangle .
\end{aligned}
$$

The experimental matrix elements for the relevant transitions in ${ }^{74} \mathrm{Kr}$ and ${ }^{76} \mathrm{Kr}$ are again summarized in Fig. 14. The resulting mixing angles and quadrupole moments are given in table XII. The system of equations has two solutions [see Eq. (2)], which are labeled + and - in the table.

Both solutions for ${ }^{74} \mathrm{Kr}$ yield a nearly maximum mixing for the two $0^{+}$states. The first solution + , however, results in a mostly oblate $2_{1}^{+}$and mostly prolate $2_{2}^{+}$state, in contradiction to the signs of the diagonal matrix elements. The large quadrupole moment for the oblate and small quadrupole moment for the prolate band of this solution are also in contradiction to the experimental results, which suggest the opposite. Therefore it seems legitimate to discard the + and adopt the - solution. The squared mixing amplitude of the prolate configuration in the ground state is thus found to be $\cos ^{2} \theta_{0}=0.48(2)$, in perfect agreement with the value $0.48(1)$ extracted from the positions of the low-spin states with respect to a rotor reference [7]. The squared mixing amplitude of $\cos ^{2} \theta_{2}=0.82(20)$ for the prolate configuration in the $2_{1}^{+}$state shows that the purity of the wave functions increases rapidly with spin. The higher-lying states of the ground-state band can be considered to have a pure prolate configuration. The quadrupole moment $Q_{0}^{\mathrm{pr}}=3.6(5) \mathrm{eb}$ agrees well with the experimental values of $Q_{0}^{t} \approx 3.1 \mathrm{eb}$ found for the transitions of the ground-state band above the $2_{1}^{+}$state (see Table X). The small value found for the quadrupole moment $Q_{0}^{\text {ob }}$ shows again that the absolute deformation for the oblate band is much smaller than for the prolate band, even though the uncertainty is rather large.

Under the assumption of the model, i.e., for a constant quadrupole moment within the intrinsic bands of pure configuration, one can use the mixing angles and quadrupole moments of Table XII to calculate further matrix elements, e.g.,

$$
\begin{aligned}
\left\langle 2_{1}\|\mathcal{M}(E 2)\| 2_{1}\right\rangle & =-\frac{5}{4} \sqrt{\frac{2}{7 \pi}}\left(Q_{0}^{\mathrm{pr}} \cos ^{2} \theta_{2}+Q_{0}^{\mathrm{ob}} \sin ^{2} \theta_{2}\right), \\
\left\langle 2_{2}\|\mathcal{M}(E 2)\| 2_{2}\right\rangle & =-\frac{5}{4} \sqrt{\frac{2}{7 \pi}}\left(Q_{0}^{\mathrm{pr}} \sin ^{2} \theta_{2}+Q_{0}^{\mathrm{ob}} \cos ^{2} \theta_{2}\right), \\
\left\langle 2_{1}\|\mathcal{M}(E 2)\| 2_{2}\right\rangle & =\frac{5}{4} \sqrt{\frac{2}{7 \pi}} \sin \theta_{2} \cos \theta_{2}\left(Q_{0}^{\mathrm{pr}}-Q_{0}^{\mathrm{ob}}\right) .
\end{aligned}
$$

The results are given in the lower part of Table XII. The matrix elements found with the second $(-)$ solution for ${ }^{74} \mathrm{Kr}$ are in reasonable agreement with the experimental values (see Tables X and XI). The opposite signs of the matrix element for the first $(+)$ solution show again the validity of adopting the solution. The two-level mixing model describes the situation in ${ }^{74} \mathrm{Kr}$ very consistently despite its simplicity and limitations. In particular, it supports the assumption of maximum mixing between a strongly prolate $\left(\beta_{2} \approx 0.4\right)$ and a weaker oblate configuration $\left(\beta_{2} \approx-0.1\right)$ for the $0^{+}$states.

TABLE XII. Mixing amplitudes for the $0^{+}$and $2^{+}$states and quadrupole moments for the bands with pure prolate and oblate configuration determined in the two-level mixing model. The columns labeled + and - correspond to the two solutions of Eq. (2). The matrix elements in the lower part were calculated from the mixing amplitudes.

\begin{tabular}{lrrrrr}
\hline \hline & \multicolumn{2}{c}{${ }^{74} \mathrm{Kr}$} & & \multicolumn{2}{c}{${ }^{76} \mathrm{Kr}$} \\
\cline { 2 - 3 } \cline { 5 - 6 } & \multicolumn{1}{c}{+} & - & & + & \multicolumn{1}{c}{-} \\
\hline $\cos ^{2}\left(\theta_{0}\right)$ & $0.52(2)$ & $0.48(2)$ & & $0.69(4)$ & $0.31(4)$ \\
$\cos ^{2}\left(\theta_{2}\right)$ & $0.18(7)$ & $0.82(20)$ & & $0.36(11)$ & $0.64(14)$ \\
$Q_{0}^{\mathrm{pr}}$ & $0.66(30)$ & $3.62(48)$ & & $2.31(31)$ & $3.48(48)$ \\
$Q_{0}^{\mathrm{ob}}$ & $-3.62(19)$ & $-0.66(86)$ & & $-3.48(41)$ & $-2.31(37)$ \\
$\left\langle 2_{1}\|\mathcal{M}(E 2)\| 2_{1}\right\rangle$ & $1.08(11)$ & $-1.08(33)$ & & $0.53(21)$ & $-0.53(25)$ \\
$\left\langle 2_{\mathrm{ex}}\|\mathcal{M}(E 2)\| 2_{\mathrm{ex}}\right\rangle^{\mathrm{a}}$ & $0.04(13)$ & $-0.04(39)$ & & $-0.09(20)$ & $0.09(24)$ \\
$\left\langle 2_{1}\|\mathcal{M}(E 2)\| 2_{\mathrm{ex}}\right\rangle^{\mathrm{a}}$ & $-0.62(11)$ & $0.62(32)$ & $-1.05(12)$ & $1.05(14)$ \\
\hline \hline
\end{tabular}

${ }^{\mathrm{a}}\left|2_{\mathrm{ex}}\right\rangle$ corresponds to $\left|2_{2}\right\rangle$ for ${ }^{74} \mathrm{Kr}$ and $\left|2_{3}\right\rangle$ for ${ }^{76} \mathrm{Kr}$. 
The two-level mixing model works less well for ${ }^{76} \mathrm{Kr}$. None of the two solutions is consistent with mostly prolate configurations for both the $0_{1}^{+}$and $2_{1}^{+}$states. The mixing angle $\theta_{0}$ of the + solution agrees well with $\cos ^{2}(\theta)=0.73(1)$ found by Bouchez et al. [7], but in this case only a small prolate amplitude is found for the $2_{1}^{+}$state. The - solution corresponds to a predominantly prolate $2_{1}^{+}$state, but then the $0_{1}^{+}$state is found predominantly oblate, in contradiction to the experimental result. The absolute values found for the quadrupole moments of the pure bands seem to be more consistent with the - solution. The signs of the diagonal matrix elements for the $2^{+}$states agree with the - solution, but the sign of the interband matrix element $\left\langle 2_{1}\|\mathcal{M}(E 2)\| 2_{3}\right\rangle$ favors the + solution, even though the absolute value from the two-level mixing model is much too large. The insufficiency of the model for ${ }^{76} \mathrm{Kr}$ is probably due to the strong coupling with the presumed quasi-gamma band, in particular between the $2_{2}^{+}$and $2_{3}^{+}$states. The different coupling of the oblate $2^{+}$ state in ${ }^{74} \mathrm{Kr}$ and ${ }^{76} \mathrm{Kr}$ is also reflected in the signs of the transitional matrix elements to the $0_{1}^{+}$and $2_{1}^{+}$states, which are opposite for ${ }^{74} \mathrm{Kr}$ and ${ }^{76} \mathrm{Kr}$. Instead of trying to include more states, e.g., in a three-band mixing model, the experimental matrix elements are compared in the following to theoretical calculations based on mean-field models, which go beyond the static approach and are able to include full configuration mixing.

\section{B. Shape coexistence in mean-field based models}

Nuclear bulk properties are well described throughout the nuclear chart by self-consistent mean-field models. To describe dynamic correlation effects and, for example, collective excitations, one has to go beyond the static mean-field approach. A technique that is well suited to describe shape coexistence is the generator coordinate method (GCM) with the quadrupole moment as generator coordinate. The method determines the configuration mixing in a variational way and provides excitation spectra and matrix elements. Selfconsistent Hartree-Fock-Bogolyubov calculations have been performed using the Gogny D1S force and the GCM approach with Gaussian overlap approximation. A five-dimensional collective Hamiltonian is used accounting for the axial and triaxial deformation and the three rotational degrees of freedom. The formalism is described in more detail in Ref. [30].

Partial results of the calculations have already been presented in Tables V, VI, X, and XI, where the spectroscopic quadrupole moments and the transition probabilities for in-band and out-of-band transitions are compared to the experimental values and to recent calculations using a similar approach by Bender et al. [21]. The latter calculations are performed with the Skyrme SLy6 force and a density-dependent pairing interaction. Bender et al. use the full GCM approach and mixing of the mean-field configurations after projection on angular momentum and particle number. The calculations are, however, restricted to axially symmetric shapes. Possible $K=$ 2 states, for example, are therefore outside the model space.

Figure 15 compares the experimental $B(E 2)$ values and quadrupole moments with the calculations as a function of

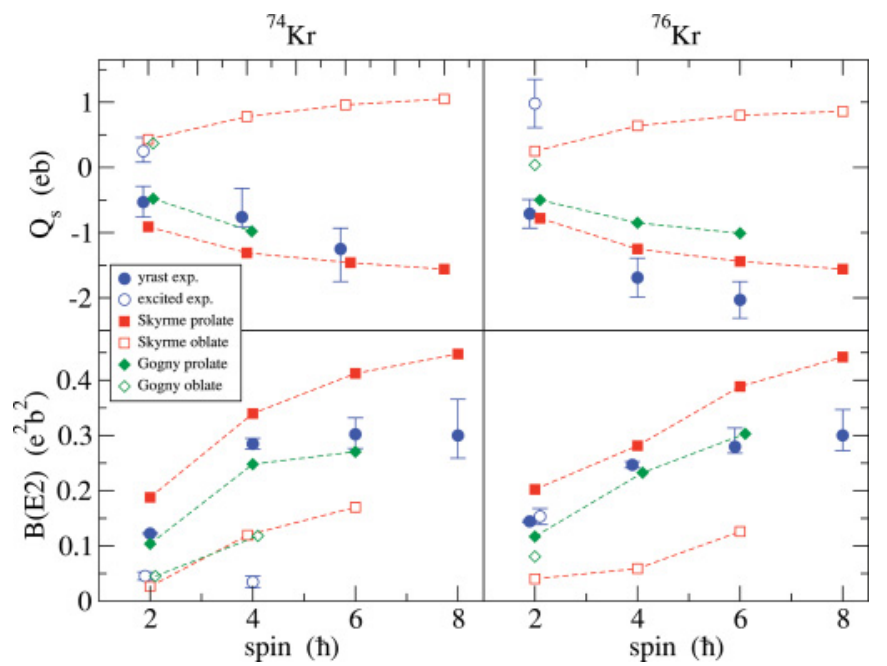

FIG. 15. (Color online) Spectroscopic quadrupole moments (top) and $B(E 2)$ values (bottom) for ${ }^{74} \mathrm{Kr}$ (left) and ${ }^{76} \mathrm{Kr}$ (right). The experimental values are compared to the configuration mixing calculations.

angular momentum. The excitation energies of the prolate and oblate states and the transition strengths between them are compared in the level schemes of Fig. 16. In the figures and the following discussion the calculations of the present work and that of Bender et al. are referred to as "Gogny" and "Skyrme," respectively. It should be noted, however, that there are other differences between the two approaches besides the effective interaction.

First it is observed that the calculation based on the Skyrme force does not reproduce the energies of the states correctly. It finds a predominantly oblate ground state and an excited prolate configuration for both ${ }^{74} \mathrm{Kr}$ and ${ }^{76} \mathrm{Kr}$ in contrast to the experimental results, which give firm evidence for the prolate character of the ground-state band. The spectra found in the Skyrme calculation are furthermore too spread out in excitation energy. It should be noted, however, that the experimental energy difference between the $0_{1}^{+}$and $0_{2}^{+}$states in ${ }^{74} \mathrm{Kr}$ of about $500 \mathrm{keV}$ is mostly due to the repulsion of the strongly mixed states, and that the intrinsic prolate and oblate configurations are therefore almost degenerate. Small deviations in energy can thus change the ordering of the prolate and oblate states in the calculation. The fact that Bender et al. find an oblate ground state also for ${ }^{76} \mathrm{Kr}$ and even for ${ }^{78} \mathrm{Kr}$ is a deficiency that was explained by the relative position of certain single-particle levels, which might not be correctly reproduced with the SLy6 effective interaction [21]. Another possible explanation is the restriction of the Skyrme calculation to axial shapes.

The five-dimensional calculation based on the Gogny force reproduces not only the excitation energies of the prolate and oblate rotational bands, but finds also a $2^{+}, 4^{+}$, and $6^{+}$state with predominant $K=2$ character in ${ }^{76} \mathrm{Kr}$ very close to the experimentally observed states of the presumed gamma-vibrational band.

The transition strengths within the prolate band and in particular the decrease toward the ground state are well reproduced by both calculations. The deformation for the states of higher spin is found somewhat too large in the Skyrme 

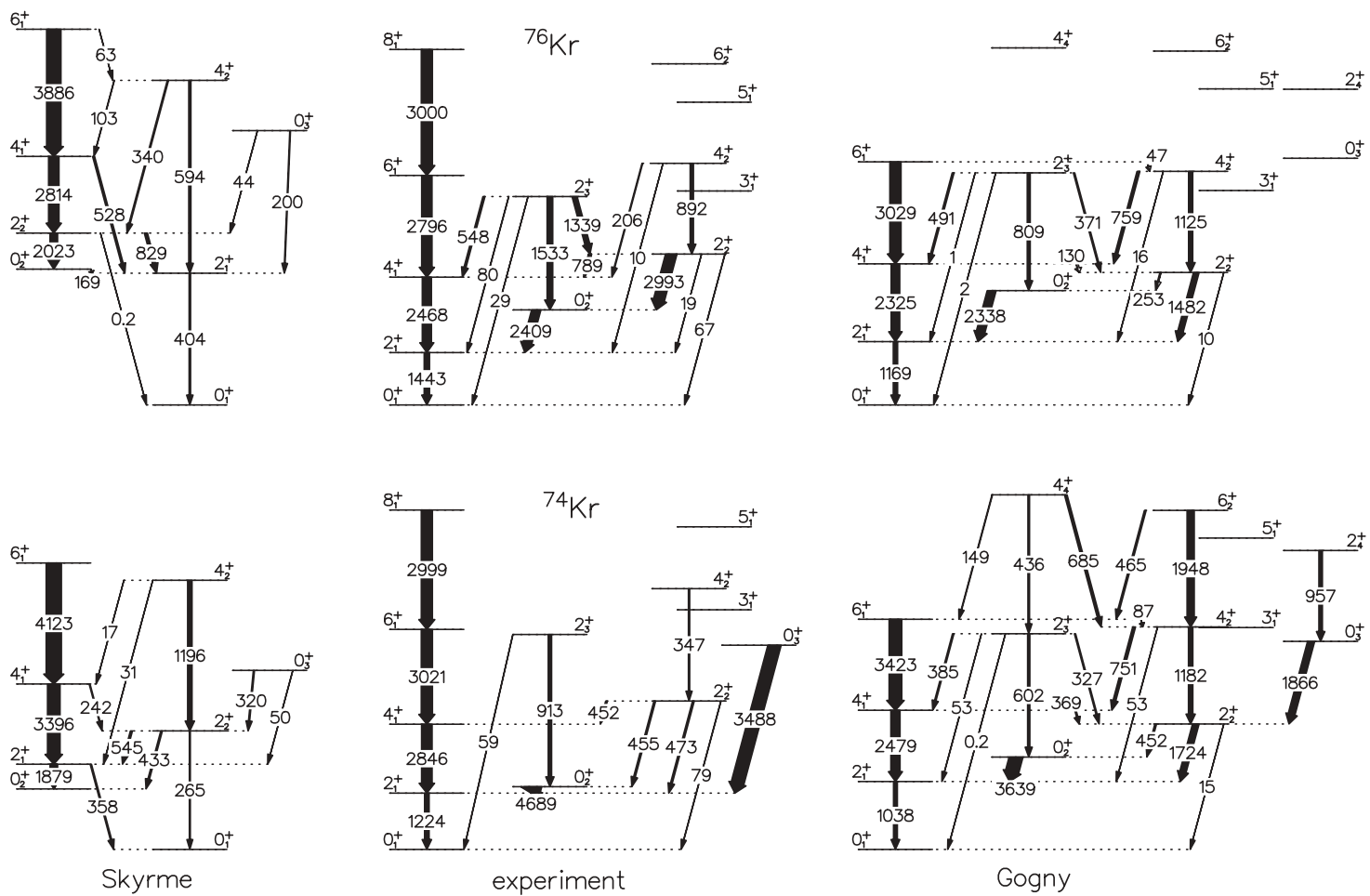

FIG. 16. Comparison between the theoretical and experimental level schemes for the oblate and prolate bands in ${ }^{76} \mathrm{Kr}$ (top) and ${ }^{74} \mathrm{Kr}$ (bottom). The excitation energies of the states are drawn to scale and the widths and labels of the arrows represent the calculated and measured $B(E 2)$ values, respectively.

calculation. The very large matrix element between the prolate $2_{1}^{+}$and the excited $0_{2}^{+}$state is well reproduced by the Gogny calculation, while it is strongly underestimated by the Skyrme calculation. It should be noted that the $B(E 2)$ values of Fig. 16 are always given in the direction of the transition and may therefore differ by a factor of $2 I_{i}+1$ in some cases where the ordering is reversed.

A grouping of the non-yrast states above the $0_{2}^{+}$state into band structures is not straightforward. The excitation energies of the $2_{2}^{+}, 3_{1}^{+}, 4_{2}^{+}, 5_{1}^{+}$, and $6_{2}^{+}$states in both isotopes are consistent with an interpretation of these sequences as gammavibrational bands. The Gogny-based calculation reproduces these sequences very well and confirms a predominant $K=2$ character of the states. In this scenario the $2_{3}^{+}$states in both isotopes can be interpreted as rotational states with oblate character built on the excited $0_{2}^{+}$states. The excitation energies and transition strengths found in the Gogny calculation are in agreement with this interpretation, as is the sign of the experimental diagonal matrix elements for the $2_{3}^{+}$states. The calculation finds strong mixing of $K=0$ and $K=2$ components for the $2_{2}^{+}$and $2_{3}^{+}$states in both isotopes, which blurs a clear classification of the states.

It is interesting to note that experimentally the $2_{2}^{+}$state couples differently in ${ }^{74} \mathrm{Kr}$ and ${ }^{76} \mathrm{Kr}$ : $\mathrm{In}{ }^{74} \mathrm{Kr}$ the state decays with equal strengths to the $2_{1}^{+}$and $0_{2}^{+}$states, whereas in ${ }^{76} \mathrm{Kr}$ it decays almost entirely to the $0_{2}^{+}$state. The different character of the $2_{2}^{+}$states in the two isotopes is also seen in the diagonal matrix elements, where opposite signs are found experimentally. If one assumes $K=2$ for the $2_{2}^{+}$ states, the negative sign of the diagonal matrix element in ${ }^{76} \mathrm{Kr}$ corresponds to oblate shape, which would explain the predominant decay to the $0_{2}^{+}$state. The positive sign found for the $2_{2}^{+}$state in ${ }^{74} \mathrm{Kr}$ corresponds to prolate shape under the assumption of $K=2$. This difference is not reproduced by the Gogny calculation, where the $2_{2}^{+}$states can be interpreted as gamma bands in the usual sense built on the ground state.

A detailed comparison of the spectroscopic quadrupole moments (see Tables VI, XI, and Fig. 15) is somewhat hampered by the rather large uncertainties of the experimental values. The spectroscopic quadrupole moments for the $4^{+}$and $6^{+}$states, in particular the very large experimental values for ${ }^{76} \mathrm{Kr}$, should be taken with care since their non-yrast partners are not known. Taking this into account, both the Gogny and the Skyrme calculations are in reasonable agreement with the experiment.

It can be concluded that configuration-mixing calculations are a valid approach to describe the shape-coexistence phenomenon. The experimental set of matrix elements in ${ }^{74} \mathrm{Kr}$ and ${ }^{76} \mathrm{Kr}$ represents a stringent test of the theoretical models. While the calculation of Bender et al. describes many global features of shape coexistence in the light krypton isotopes correctly, certain details are not reproduced, in particular the ordering of the prolate and oblate states. The agreement with the Gogny calculation, on the other hand, is remarkable. This suggests that it is important to include the triaxial degree of freedom in the calculations. This result will be discussed in more detail in a forthcoming article from the theoretical perspective [31]. 


\section{Rotational invariants and the sum-rule method}

Finally, the matrix elements are analyzed using a modelindependent description of the nuclear shape in terms of global quadrupole-deformation parameters. This can be done by constructing rotational invariants of zero-coupled products of the $E 2$ operator, which can be linked to the deformation parameters in the intrinsic frame of the nucleus $[8,16]$.

The electric quadrupole operator $E(\lambda=2, \mu)$ is a spherical tensor that can be parameterized in the principal axes frame using the two parameters $Q$ and $\delta$ as:

$$
\begin{aligned}
& E(2,0)=Q \cos (\delta) \\
& E(2,1)=E(2,-1)=0 \\
& E(2,2)=E(2,-2)=\frac{1}{\sqrt{2}} Q \sin (\delta) .
\end{aligned}
$$

The parameter $Q$ measures the overall deformation and is equivalent to the elongation parameter $\beta$ in the Bohr model, whereas $\delta$ is related to the triaxiality parameter $\gamma$. The simplest zero-coupled products of the $E 2$ operator can then be written as:

$$
\begin{aligned}
\left\langle I\left\|[E 2 \times E 2]_{0}\right\| I\right\rangle & =\frac{Q^{2}}{\sqrt{5}}, \\
\left\langle I\left\|\left\{[E 2 \times E 2]_{2} \times E 2\right\}_{0}\right\| I\right\rangle & =-\sqrt{\frac{2}{35}} Q^{3} \cos (3 \delta) .
\end{aligned}
$$

The E2 invariants can be expressed in the laboratory frame by an expansion over all possible intermediate states using Wigner's $6 j$ symbols:

$$
\begin{aligned}
&\left\langle I_{i} \|[\right.\left.E 2 \times E 2]_{0} \| I_{i}\right\rangle \\
&= \frac{(-1)^{2 I_{i}}}{\sqrt{2 I_{i}+1}} \sum_{j}\left\langle I_{i}\|\mathcal{M}(E 2)\| I_{j}\right\rangle\left\langle I_{j}\|\mathcal{M}(E 2)\| I_{i}\right\rangle \\
& \times\left\{\begin{array}{rrr}
2 & 2 & 0 \\
I_{i} & I_{i} & I_{j}
\end{array}\right\}, \\
&\left\langle I_{i}\left\|\left\{[E 2 \times E 2]_{2} \times E 2\right\}_{0}\right\| I_{i}\right\rangle \\
&=\frac{1}{2 I_{i}+1} \sum_{j, k}\left\langle I_{i}\|\mathcal{M}(E 2)\| I_{j}\right\rangle \\
& \quad \times\left\langle I_{j}\|\mathcal{M}(E 2)\| I_{k}\right\rangle\left\langle I_{k}\|\mathcal{M}(E 2)\| I_{i}\right\rangle\left\{\begin{array}{lll}
2 & 2 & 2 \\
I_{i} & I_{j} & I_{k}
\end{array}\right\} .
\end{aligned}
$$

If the relevant matrix elements are experimentally known, the deformation parameters can be determined in a modelindependent way by summing over all closed loops of E2 matrix elements, including diagonal ones, connected to a specific state. This method is particularly useful to attribute shape parameters to $0^{+}$states, which are not directly accessible in the laboratory frame. Besides statistical errors of the E2 matrix elements, an additional systematic error comes from an incomplete knowledge of the matrix elements. Any loop that is not considered in the sum will reduce the deformation parameter $Q^{2}$ and may change the triaxiality parameter $\cos (3 \delta)$ depending on the relative signs of the
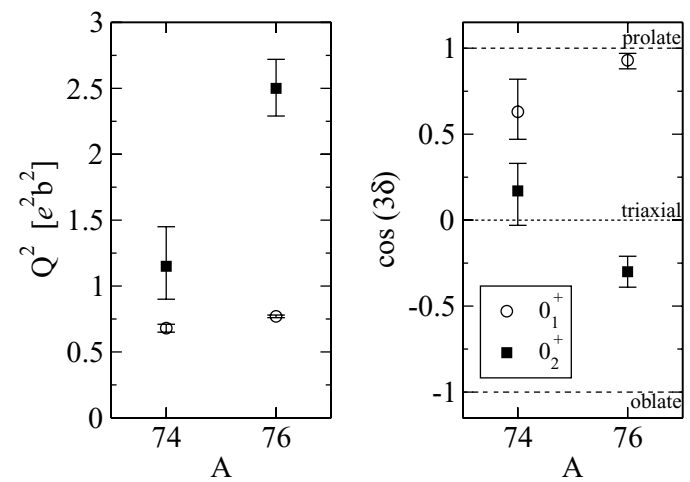

FIG. 17. Quadrupole deformation parameters obtained by the sum-rule method for the $0^{+}$states in ${ }^{74} \mathrm{Kr}$ and ${ }^{76} \mathrm{Kr}$.

unknown matrix elements. The analysis of the rotational invariants in the present case of ${ }^{74} \mathrm{Kr}$ and ${ }^{76} \mathrm{Kr}$ has therefore been restricted to the $0^{+}$states, so that only the transitional matrix elements $\left\langle 0_{i}\|\mathcal{M}(E 2)\| 2_{j}\right\rangle$ and $\left\langle 2_{i}\|\mathcal{M}(E 2)\| 2_{j}\right\rangle$ and the diagonal matrix elements $\left\langle 2_{i}\|\mathcal{M}(E 2)\| 2_{i}\right\rangle$ have to be considered. In deformed nuclei most of the $E 2$ strength is exhausted by the first rotational $2^{+}$state. Due to the shape coexistence and configuration mixing also the excited $2^{+}$states contribute significantly to the shape parameters of the $0^{+}$states in the krypton isotopes. Extending the analysis to states of higher spin seems unreliable because the set of matrix elements cannot be considered to be complete for higher-spin states.

The resulting quadrupole deformation parameters for the first and second $0^{+}$states in ${ }^{74} \mathrm{Kr}$ and ${ }^{76} \mathrm{Kr}$ are presented in Fig. 17. The $Q^{2}$ parameter is very similar for the ground states in ${ }^{74} \mathrm{Kr}$ and ${ }^{76} \mathrm{Kr}$. It seems surprising that in ${ }^{74} \mathrm{Kr}$ the deformation parameter for the excited oblate $0_{2}^{+}$state is larger than that of the prolate ground state, even though the absolute value of the experimental quadrupole moment is larger for the prolate $2_{1}^{+}$than for either of the non-yrast $2^{+}$states. The difference between the ground state and excited $0_{2}^{+}$state is even more pronounced in ${ }^{76} \mathrm{Kr}$. The extremely large $Q^{2}$ value of the $0_{2}^{+}$state is related to the strong coupling of this state to all three $2^{+}$states, in particular to the $2_{2}^{+}$state. It is interesting to note in this context that the experimental quadrupole moment of the oblate $2_{3}^{+}$state is indeed very large, and also the analysis in the two-band mixing model suggests a rather large oblate deformation, so that there seems to be experimental evidence for an increase in oblate deformation from ${ }^{74} \mathrm{Kr}$ to ${ }^{76} \mathrm{Kr}$.

The parameter $\cos (3 \delta)$ suggests an almost purely prolate shape for the ground state of ${ }^{76} \mathrm{Kr}$ and a mostly prolate shape with some triaxiality for the ground state of ${ }^{74} \mathrm{Kr}$. An oblate triaxial shape is found for the excited $0_{2}^{+}$state in ${ }^{76} \mathrm{Kr}$ and maximum triaxiality for the $0_{2}^{+}$state in ${ }^{74} \mathrm{Kr}$. The results found for the triaxiality parameter $\cos (3 \delta)$ are in qualitative agreement with the findings of the two-band mixing model. The mixing of prolate and oblate configurations leads to a certain triaxiality of the $0^{+}$states. In the case of ${ }^{74} \mathrm{Kr}$ the maximum mixing between a large prolate and smaller oblate deformation could introduce a slight triaxiality in the ground state, but affect the shape of the $0_{2}^{+}$state much stronger, resulting in a shape with large triaxiality. The results again 
confirm the importance of the triaxial degree of freedom for the description of the shape coexistence phenomenon.

\section{Conclusions}

The discussion in the previous sections has shown that a consistent description of the complex shape coexistence and interpretation of the experimental data can be achieved by sophisticated theoretical calculations going beyond the static mean-field approach by including the mixing of many basis states. It is found that the structure of the low-lying states in the light krypton isotopes is dominated by the coexistence of prolate and oblate configurations. The phenomenological two band-mixing model gives some insight into the mixing of the wave functions, but it is clearly too simple to describe all experimental data. The formalism of quadrupole sum rules is useful to derive shape parameters in a model-independent way, in particular for $0^{+}$states. However, the requirement of complete sets of matrix elements limits the applicability of the method.

Configuration-mixing calculations based on mean-field models and the generator-coordinate method offer the possibility to describe the physical states, including transition strengths and spectroscopic quadrupole moments. The obtained agreement with the experimental data is satisfying, keeping in mind that there are no free parameters to adjust, and that the effective interactions are derived globally. From the results it seems clear that the triaxial degree of freedom must be included into such calculations. To further conclude about the effective interaction, it would be instructive to perform configuration-mixing calculations with the Gogny interaction while enforcing axial symmetry, and, on a longerterm perspective, to include triaxiality in the approach based on the Skyrme interaction.

\section{SUMMARY}

Shape coexistence in the light krypton isotopes was studied in two low-energy Coulomb excitation experiments using the radioactive ${ }^{74} \mathrm{Kr}$ and ${ }^{76} \mathrm{Kr}$ beams of the SPIRAL facility. Transitional and diagonal matrix elements of low-lying states in ${ }^{74} \mathrm{Kr}$ and ${ }^{76} \mathrm{Kr}$ were extracted from the observed $\gamma$-ray yields. The transition strengths are in good agreement with the results of a recent lifetime measurement. Using the precise lifetimes as additional input in the analysis of the Coulomb excitation data enhanced the sensitivity to the reorientation effect and allowed extracting spectroscopic quadrupole moments for several excited states. The negative diagonal matrix elements of the states in the ground-state bands of both isotopes under study prove their prolate character. The excited $2_{2}^{+}$and $2_{3}^{+}$states are interpreted as gamma-vibrational and oblate rotational states, respectively. However, a clear classification of the states is difficult due to the mixing of prolate and oblate configurations, on one hand, and $K=0$ and $K=2$ configurations, on the other hand.

The matrix elements were interpreted in a phenomenological two-band mixing model. The results confirm the earlier finding of maximum mixing between prolate and oblate configurations in the wave functions of the $0^{+}$states in ${ }^{74} \mathrm{Kr}$. The purity of the wave functions increases rapidly with spin, explaining the increase in the transition strength and at the same time the very strong interband transitions between the different $2^{+}$and $0^{+}$states. However, the model fails to describe consistently the band mixing in ${ }^{76} \mathrm{Kr}$, probably due to the strong coupling with the presumed gamma-vibrational band. The quadrupole sum rule formalism was applied to derive shape parameters for the $0^{+}$states.

The transition strengths and quadrupole moments are compared to recent theoretical calculations going beyond the static mean-field approach. The confrontation of the calculations with the large set of experimental matrix elements represents a stringent test of the models and effective interactions. The results emphasize the importance of including the triaxial degree of freedom to describe the shape coexistence in the light krypton isotopes.

The present work represents the first direct experimental proof of the proposed shape coexistence scenario, and the reorientation effect has been exploited for the first time with a radioactive ion beam. The ground-state shape of the $N=$ $Z$ nucleus ${ }^{72} \mathrm{Kr}$ is expected to be dominated by the oblate configuration. The relatively small $B\left(E 2 ; 0_{1}^{+} \rightarrow 2_{1}^{+}\right)$value found for ${ }^{72} \mathrm{Kr}$ in an intermediate-energy Coulomb excitation experiment has been interpreted as favoring an oblate shape [32], but cannot prove the inversion of the ground-state shape for ${ }^{72} \mathrm{Kr}$. The measurement of quadrupole moments in ${ }^{72} \mathrm{Kr}$ remains a challenge for future low-energy Coulomb excitation experiments.

\section{ACKNOWLEDGMENTS}

The authors would like to thank the technical staff at GANIL for excellent support. Stimulating discussions with M. Bender, P.-H. Heenen, and J. Srebrny are gratefully acknowledged. This work has been in part supported by the European Community FP6-Structuring the ERA - Integrated Infrastructure Initiative - contract EURONS no. RII3-CT2004-506065 and by EPSRC (UK).
[1] A. Bohr and B. R. Mottelson, Nuclear Structure (W. A. Benjamin, Reading, 1975).

[2] N. Tajima and N. Suzuki, Phys. Rev. C 64, 037301 (2001).

[3] R. Bengtsson in Nuclear Structure of the Zirconium Region, edited by J. Eberth, R. M. Meyer, and K. Siestemich (Springer, Berlin, 1988).

[4] R. B. Piercey, J. H. Hamilton, R. Soundranayagam, A. V. Ramayya, C. F. Maguire, X.-J. Sun, Z. Z. Zhao, R. L. Robinson,
H. J. Kim, S. Frauendorf, J. Döring, L. Funke, G. Winter, J. Roth, L. Cleemann, J. Eberth, W. Neumann, J. C. Wells, J. Lin, A. C. Rester, and H. K. Carter, Phys. Rev. Lett. 47, 1514 (1981).

[5] C. Chandler, P. H. Regan, C. J. Pearson, B. Blank, A. M. Bruce, W. N. Catford, N. Curtis, S. Czajkowski, W. Gelletly, R. Grzywacz, Z. Janas, M. Lewitowicz, C. Marchand, N. A. Orr, R. D. Page, A. Petrovici, A. T. Reed, M. G. Saint-Laurent, 
S. M. Vincent, R. Wadsworth, D. D. Warner, and J. S. Winfield, Phys. Rev. C 56, R2924 (1997).

[6] F. Becker, W. Korten, F. Hannachi, P. Paris, N. Buforn, C. Chandler, M. Houry, H. Hübel, A. Jansen, Y. Le Coz, C. F. Liang, A. Lopez-Martens, R. Lucas, E. Mergel, P. H. Regan, G. Schönwasser, and Ch. Theisen, Eur. Phys. J. A 4, 103 (1999).

[7] E. Bouchez, I. Matea, W. Korten, F. Becker, B. Blank, C. Borcea, A. Buta, A. Emsallem, G. de France, J. Genevey, F. Hannachi, K. Hauschild, A. Hürstel, Y. Le Coz, M. Lewitowicz, R. Lucas, F. Negoita, F. de Oliveira Santos, D. Pantelica, J. Pinston, P. Rahkila, M. Rejmund, M. Stanoiu, and Ch. Theisen, Phys. Rev. Lett. 90, 082502 (2003).

[8] D. Cline, Annu. Rev. Nucl. Part. Sci. 36, 683 (1986).

[9] K. Alder and A. Winther, Electromagnetic Excitation, Theory of Coulomb Excitation with Heavy Ions (North-Holland, Amsterdam, 1975).

[10] W. Mittig, J. Phys. G: Nucl. Part. Phys. 24, 1331 (1998).

[11] P. M. S. Lesser, D. Cline, Philip Goode, and R. N. Horoshko, Nucl. Phys. A190, 597 (1972).

[12] F. Azaiez, G. de France, R. Julin, P. J. Nolan, B. M. Nyako, P. M. Walker, J. Simpson, J. Fouan, W. Korten, G. Sletten, J. Gerl, and the EXOGAM Collaboration, Acta Phys. Hung. A 11, 159 (2000).

[13] B. Singh, Nucl. Data Sheets 74, 63 (1995).

[14] R. B. Piercey, A. V. Ramayya, J. H. Hamilton, X. J. Sun, Z. Z. Zhao, R. L. Robinson, H. J. Kim, and John C. Wells, Phys. Rev. C 25, 1941 (1982).

[15] A. Piechaczek, E. F. Zganjar, G. C. Ball, P. Bricault, J. M. D’Auria, J. C. Hardy, D. F. Hodgson, V. Iacob, P. Klages, W. D. Kulp, J. R. Leslie, M. Lipoglavsek, J. A. Macdonald, H.-B. Mak, D. M. Moltz, G. Savard, J. von Schwarzenberg, C. E. Svensson, I. S. Towner, and J. L. Wood, Phys. Rev. C 67, 051305(R) (2003).

[16] T. Czosnyka, D. Cline, and C. Y. Wu, Bull. Am. Phys. Soc. 28, 745 (1983).

[17] A. Giannatiempo, A. Perego, P. Sona, A. Nannini, H. Mach, B. Fogelberg, M. J. G. Borge, O. Tengblad, L. M. Fraile, A. J. Aas, K. Gulda, Phys. Rev. C 72, 044308 (2005).

[18] B. Wörmann, K. P. Lieb, R. Diller, L. Lühmann, J. Keinonen, L. Cleemann, and J. Eberth, Nucl. Phys. A431, 170 (1984).

[19] J. J. Valiente-Dobón, C. E. Svensson, C. D. O’Leary, I. Ragnarsson, C. Andreoiu, D. E. Appelbe, R. A. E. Austin, G. C. Ball, J. A. Cameron, M. P. Carpenter, R. M. Clark, M. Cromaz, D. Dashdorj, P. Fallon, P. Finlay, S. J. Freeman, P. E. Garrett, A. Görgen, G. F. Grinyer, D. F. Hodgson, B. Hyland,
D. Jenkins, F. Johnston-Theasby, P. Joshi, N. S. Kelsall, A. O. Macchiavelli, F. Moore, G. Mukherjee, A. A. Phillips, W. Reviol, D. Sarantites, M. A. Schumaker, D. Seweryniak, M. B. Smith, J. C. Waddington, R. Wadsworth, D. Ward, and S. J. Williams, Phys. Rev. C 71, 034311 (2005).

[20] A. Görgen, E. Clément, A. Chatillon, A. Dewald, W. Korten, Y. Le Coz, N. Marginean, B. Melon, R. Menegazzo, O. Möller, Ch. Theisen, D. Tonev, C. A. Ur, and K. O. Zell, Eur. Phys. J. A 26, 153 (2005).

[21] M. Bender, P. Bonche, and P.-H. Heenen, Phys. Rev. C 74, 024312 (2006).

[22] T. Czosnyka, D. Cline, and C. Y. Wu, GOSIA manual, University of Rochester, unpublished.

[23] F. Bosch and H. Spehl, Z. Phys. A 280, 329 (1977).

[24] R. Brenn, H. Spehl, A. Weckherlin, H. A. Doubt, and G. van Middelkoop, Z. Phys. A 281, 219 (1977).

[25] G. Kumbartzki, J. R. Cooper, N. Benczer-Koller, K. Hiles, T. J. Mertzimekis, M. J. Taylor, K.-H. Speidel, P. Maier-Komor, L. Bernstein, M. A. McMahan, L. Phair, J. Powell, and D. Wutte, Phys. Lett. B591, 213 (2004).

[26] S. L. Tabor, P. D. Cottle, J. W. Holcomb, T. D. Johnson, P. C. Womble, S. G. Buccino, and F. E. Durham, Phys. Rev. C 41, 2658 (1990).

[27] J. Roth, L. Cleemann, J. Eberth, T. Heck, W. Neumann, M. Nolte, R. B. Piercey, A. V. Ramayya, and J. H. Hamilton, J. Phys. G: Nucl. Phys. 10, L25 (1984).

[28] J. Heese, D. J. Blumenthal, A. A. Chishti, P. Chowdhury, B. Crowell, P. J. Ennis, C. J. Lister, and Ch. Winter, Phys. Rev. C 43, R921 (1991).

[29] A. Algora, G. de Angelis, F. Brandolini, R. Wyss, A. Gadea, E. Farnea, W. Gelletly, S. Lunardi, D. Bazzacco, C. Fahlander, A. Aprahamian, F. Becker, P. G. Bizzeti, A. Bizzeti-Sona, D. de Acuna, M. De Poli, J. Eberth, D. Foltescu, S. M. Lenzi, T. Martinez, D. R. Napoli, P. Pavan, C. M. Petrache, C. Rossi Alvarez, D. Rudolph, B. Rubio, S. Skoda, P. Spolaore, R. Menegazzo, H. G. Thomas, and C. A. Ur, Phys. Rev. C 61, 031303(R) (2000).

[30] J. Libert, M. Girod, and J.-P. Delaroche, Phys. Rev. C 60, 054301 (1999).

[31] J.-P. Delaroche, M. Girod et al., in preparation.

[32] A. Gade, D. Bazin, A. Becerril, C. M. Campbell, J. M. Cook, D. J. Dean, D.-C. Dinca, T. Glasmacher, G. W. Hitt, M. E. Howard, W. F. Mueller, H. Olliver, J. R. Terry, and K. Yoneda, Phys. Rev. Lett. 95, 022502 (2005). 